\title{
Real-Time Data Compression Using a Fixed Huffman Encoding Scheme
}

\author{
R. E. Kidner
}

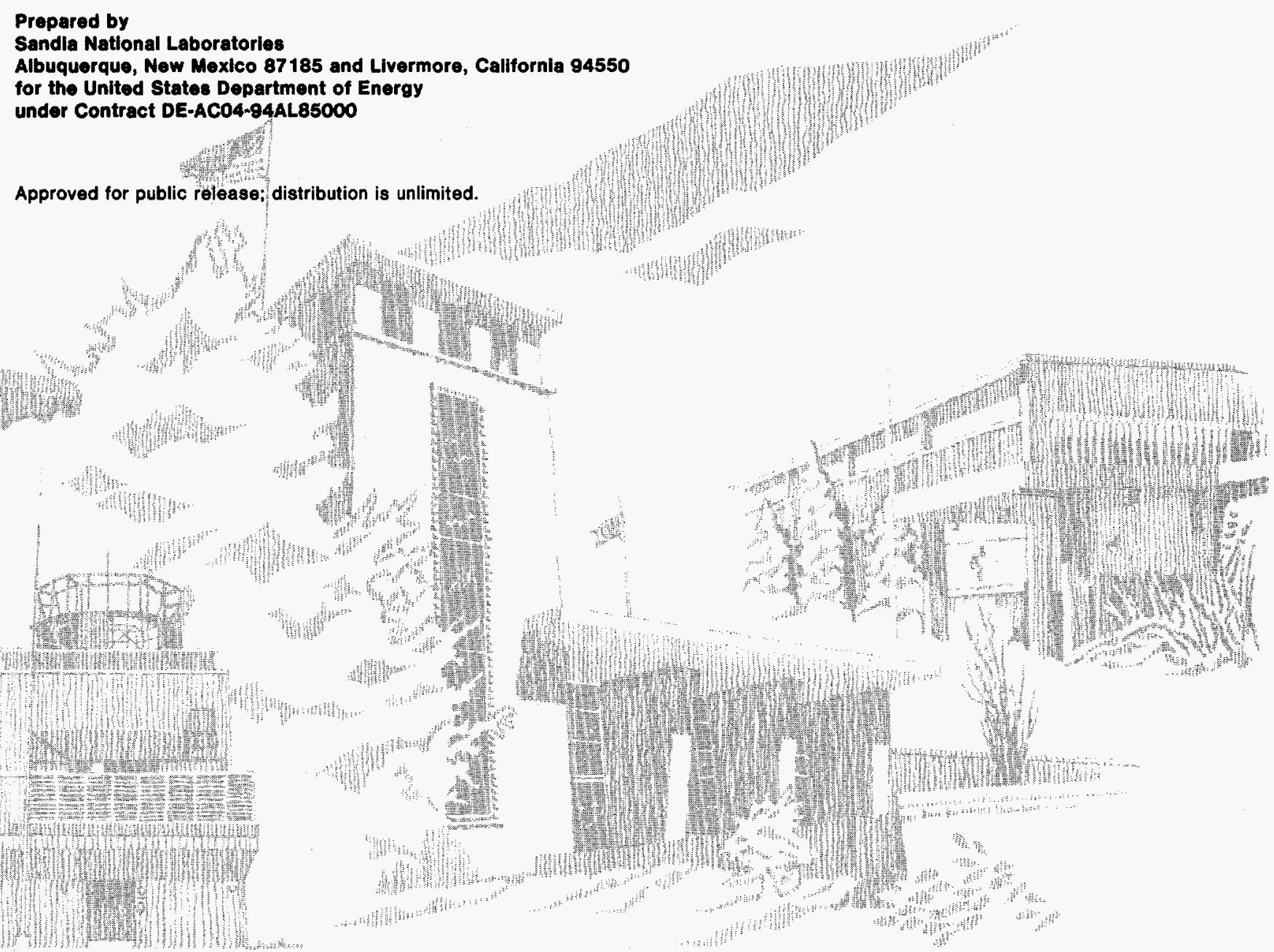


Issued by Sandia National Laboratories, operated for the United States Department of Energy by Sandia Corporation.

NOTICE: This report was prepared as an account of work sponsored by an agency of the United States Government. Neither the United States Government nor any agency thereof, nor any of their employees, nor any of their contractors, subcontractors, or their employees, makes any warranty, express or implied, or assumes any legal liability or responsibility for the accuracy, completeness, or usefulness of any information, apparatus, product, or process disclosed, or represents that its use would not infringe privately owned rights. Reference herein to any specific commercial product, process, or service by trade name, trademark, manufacturer, or otherwise, does not necessarily constitute or imply its endorsement, recommendation, or favoring by the United States Government, any agency thereof or any of their contractors or subcontractors. The views and opinions expressed herein do not necessarily state or reflect those of the United States Government, any agency thereof or any of their contractors.

Printed in the United States of America. This report has been reproduced directly from the best available copy.

Available to DOE and DOE contractors from

Office of Scientific and Technical Information

PO Box 62

Oak Ridge, TN 37831

Prices available from (615) 576-8401, FTS 626-8401

Available to the public from

National Technical Information Service

US Department of Commerce

5285 Port Royal RD

Springfield, VA 22161

NTIS price codes

Printed copy: A03

Microfiche copy: A01 


\section{DISCLAIMER}

Portions of this document may be illegible in electronic image products. Images are produced from the best available original document. 


\title{
REAL-TIME DATA COMPRESSION USING A FIXED HUFFMAN ENCODING SCHEME
}

\author{
R. E. Kidner \\ Telemetry Technology Development Department \\ Sandia National Laboratories \\ Albuquerque, NM 87185
}

\begin{abstract}
$\underline{\text { Abstract }}$
A common limitation to performance in data acquisition systems is storage of the collected data. Compressing the data would increase the amount of data that could be stored. However, most compression routines require that the data be collected and analyzed before compression is performed. Also, these compression routines often store the information required for decompression along with the data, thus decreasing the storage available for data. One solution to this problem is to create an encoding tree known to both the encoder and the decoder based on apriori knowledge of the data. Once the tree is created, optimal encoding schemes such as the Huffman algorithm may be used on the data as it is being collected. In this way the data is compressed as each byte is received and there is no overhead associated with storing decompression data. In this paper the idea of using a fixed Huffman tree is explored and the results are compared to a defacto standard in data compression, PKZIP.
\end{abstract}

DISTRIBUTION OF THIS DOCUMENT IS UNLIMTED 


\section{Acknowledgments}

The author wishes to acknowledge Bryan Cook for his assistance in this project. The time and effort he spent testing many of the concepts contained within this report is greatly appreciated. 


\section{CONTENTS}

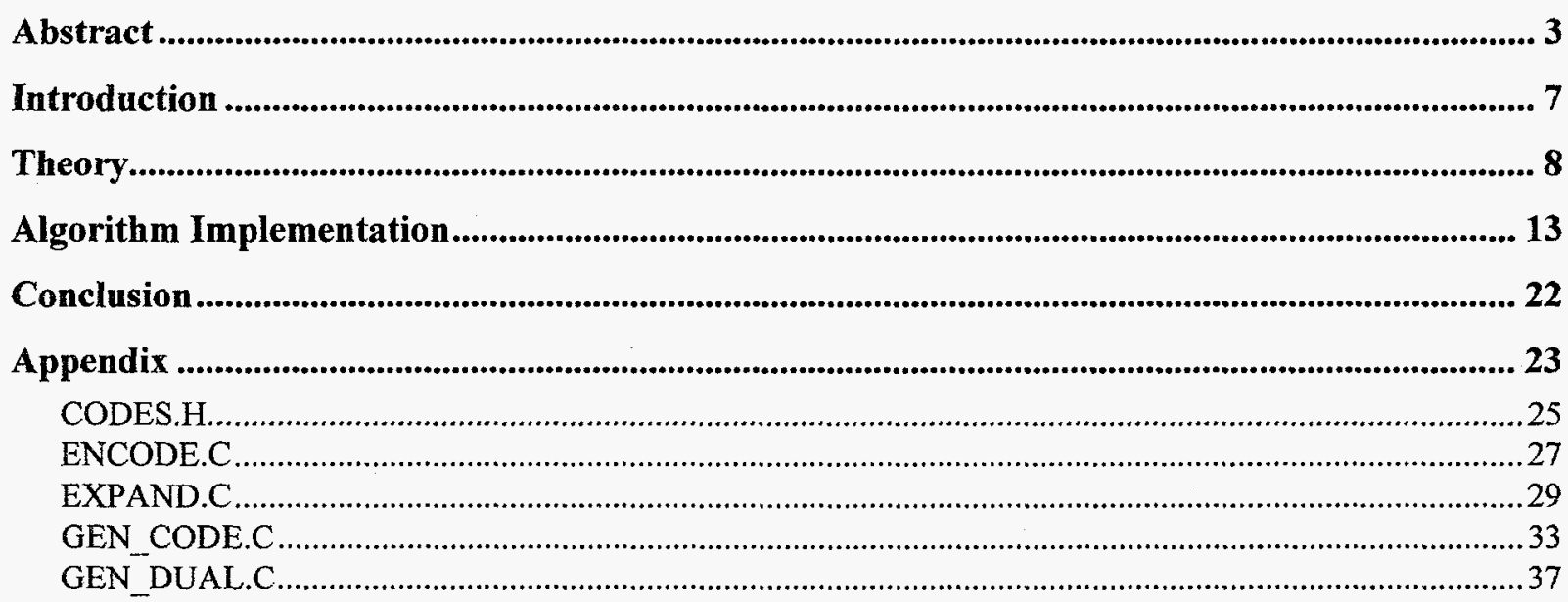

\section{FIGURES}

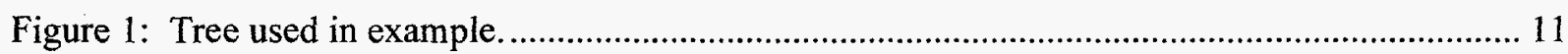

Figure 2: Composite data used in the algorithm development. .............................................. 13

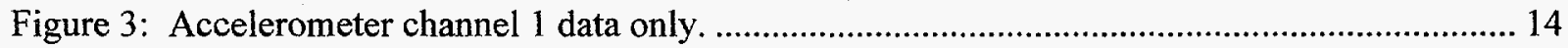

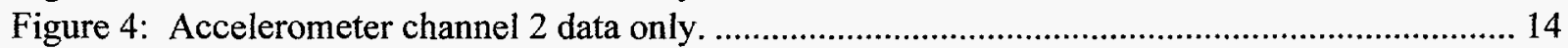

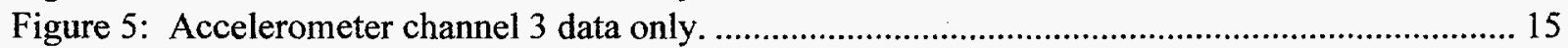

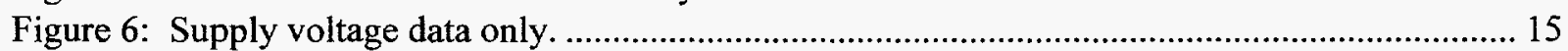

Figure 7: Three Gaussian curves with means of 123 and sigmas of 5,10 , and $15 \ldots \ldots \ldots \ldots \ldots \ldots \ldots \ldots . . . . . . . . . .17$

Figure 8: Three Gaussian curves with means of 120,123 , and 126 and sigmas of $10 \ldots \ldots \ldots \ldots \ldots \ldots . . \ldots 18$

Figure 9: Gaussian curve yielding the greatest compression of the 5 on channel 2 ..................... 19

\section{TABLES}

Table 1: Number of occurrences for each data in the example phrase. ............................................ 8

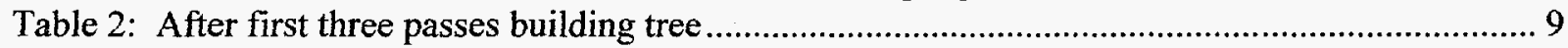

Table 3: Tree after collection of 2 occurrence nodes..................................................................... 9

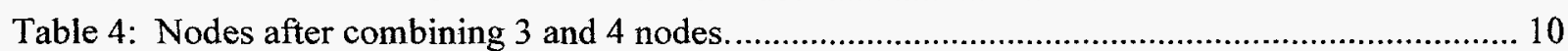

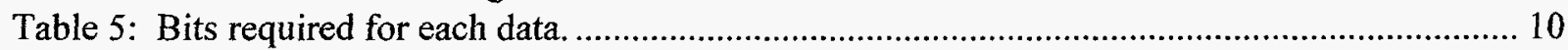

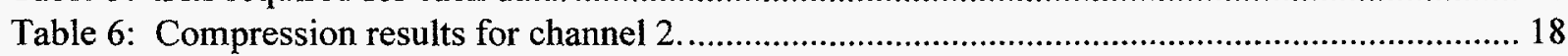

Table 7: Compression results for composite data using a single curve ....................................... 20

Table 8: Comparison of single and dual function compression on the composite data set................20

Table 9: Channels 1, 2, 3 and 4 compression results using a single curve. .......................................2 20

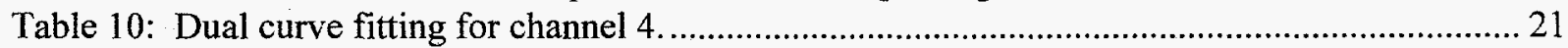

Table 11: Compression of channels 1, 2, and 3 using a single curve. ......................................... 21

Table 12: Compression results comparison using a single Gaussian function.................................. 22

Table 13: Comparisons with PKZIP when using two functions to generate the tree. ...................... 22 


\section{REAL-TIME DATA COMPRESSION USING A FIXED HUFFMAN ENCODING SCHEME}

\section{Introduction}

A common limitation to performance in data acquisition systems is storage of the collected data. High speed systems are often limited to less than a second of data collection due to the volume of data collected. Long term systems must limit the speed at which they collect data in order to continue collection over the days or weeks of an experiment. In either extreme, compressing the resulting data before storage would greatly enhance the memory performance of the system. Unfortunately, most optimal compression schemes require most, if not all, the data be collected before compression is performed. This is because most compression routines compute statistics of the complete data set, and then construct the encoding tree based on these statistics. Only after the tree is constructed may the data be compressed. Not only is the compression not done in real-time, but there is some penalty associated with storing the encoding tree. A solution to this problem is to create an encoding tree known to both the encoder and the decoder based on apriori knowledge of the data. Once the tree is created, optimal encoding schemes such as the Huffman algorithm may be used on the data as it is being collected.

In this paper, a compression algorithm is developed to perform real-time compression on data as it is gathered. The algorithm is based on the Huffman encoding scheme, but the tree used is generated off line before the data acquisition system is used. The data used to test the algorithm is actual data gathered during an environmental test of a moving railcar. The results of the algorithm using the railcar data are compared to the results of PKZIP, version 2. 


\section{Theory}

A widely accepted optimal encoding scheme is the Huffman encoding scheme. This scheme is based on statistics gathered on the data prior to compression. Using this statistical data, an encoding tree is constructed. The tree is constructed such that the most often occurring data require the least number of branches to arrive at its leaf node. Since each branch requires one binary bit to determine which branch to negotiate, the most often occurring data will require the fewest number of bits to uniquely encode the data. Conversely, the data that seldom occur are assigned nodes of the tree which require the most bits to negotiate the tree. As an example we will build a tree to compress the following phrase:

\section{REAL-TIME DATA COMPRESSION}

If the phrase were stored as ASCII characters, it would require 26 bytes (208 bits) to store the 26 data symbols in the phrase.

First the 'file' is scanned and the number of occurrences of each data is recorded. Table 1 lists the data and the number of occurrences for each.

\begin{tabular}{|l|l||l|l||l|l|}
\hline Data & Occurrences & Data & Occurrences & Data & Occurrences \\
\hline \hline A & 3 & L & 1 & R & 2 \\
\hline C & 1 & M & 2 & S & 2 \\
\hline D & 1 & N & 1 & T & 2 \\
\hline E & 3 & O & 2 & - & 1 \\
\hline I & 2 & P & 1 & space & 2 \\
\hline
\end{tabular}

Table 1: Number of occurrences for each data in the example phrase.

The tree is built from the leaves (the character data in this case) toward the root. Starting from these leaves, the least occurring data, as defined in Table 1 , are connected by a parent node, one child pair at a time. As each pair is connected, the newly created parent node assumes the value of the sum of its two children. In this way, each parent node reflects the number of data occurrences below it. Then the table is scanned again, looking for the lowest valued nodes (child or parent). In our example, there are 6 characters that only occur once. The order in which ties are resolved is not important, so we will choose the order in which they appear in Table 1. Therefore leaves ' $C$ ' and ' $D$ ' are combined into a parent node with a value of 2 . The data ' $C$ ' and ' $D$ ' are not used again. To chose the next pair of data, Table 1 is scanned again. 
This time ' $\mathrm{L}$ ' and ' $N$ ' are combined. Likewise ' $\mathrm{P}$ ' and '-' are combined. After these three operation there are three fewer nodes. Table 2 shows the current value of our tree.

\begin{tabular}{|l|l||l|l||l|l|}
\hline Node & Value & Node & Value & Node & Value \\
\hline \hline A & 3 & LN & 2 & R & 2 \\
\hline CD & 2 & M & 2 & S & 2 \\
\hline & & & & T & 2 \\
\hline E & 3 & O & 2 & & \\
\hline I & 2 & P- & 2 & space & 2 \\
\hline
\end{tabular}

Table 2: After first three passes building tree

The next least occurring nodes occur 2 times each. Table 2 is then scanned, combining all nodes occurring 2 times each. The 7 pairs of nodes are combined and the result is Table 3 .

\begin{tabular}{|c|c|c|c|c|c|}
\hline Node & Value & Node & Value & Node & Value \\
\hline $\bar{A}$ & 3 & $\mathrm{LMN}$ & 4 & $\mathrm{RS}$ & 4 \\
\hline \multirow[t]{2}{*}{$\mathrm{CDI}$} & 4 & & & & \\
\hline & & & & Tspace & 4 \\
\hline$E$ & 3 & OP- & 4 & & \\
\hline & & & & & \\
\hline
\end{tabular}

Table 3: Tree after collection of 2 occurrence nodes.

The next combination of nodes occur on the two 3 occurrence nodes. This is followed by the five 4 occurrence nodes. Since there are an odd number of 4 occurrence nodes, one will be left alone until it is combined with the new node of value 6 . Table 4 shows the result of these operations. 


\begin{tabular}{|l|l||l|l||l|l|}
\hline Node & Value & Node & Value & Node & Value \\
\hline \hline AE & 6 & & & & \\
\hline CDILMN & 8 & & & & \\
\hline & & & & Tspace & 4 \\
\hline & & & & & \\
\hline
\end{tabular}

Table 4: Nodes after combining 3 and 4 nodes.

Again the table is scanned, this time combining the nodes with values 4 and 6 . Then the nodes with value 8 are combined. Finally the new nodes with value 10 and 16 are combined into a single root node. In this way, all data found in the file are connected in the tree under one root node. Figure 1 shows the tree constructed from the example phrase. The circles on level 1 are the leaves and include the data and the number of occurrences of the data. The circles in the other levels show the composite value of their children. These are used to determine which nodes are combined at each level. The numbers along side the connecting lines are binary bits used to navigate each branch. For example, by following the path to the $\mathrm{R}$ leaf we see the code value for $\mathrm{R}$ is 0110 . This tree is used in the forward direction (root node toward the leaves) when decoding data and is used in reverse direction when encoding. Table 5 shows how many bits each data requires to encode it using this example tree. Notice this tree is not a straight binary tree. That is, a straight binary tree would only require a maximum code of 4 bits to encode 15 unique data. The Huffman tree is skewed to minimize the number of bits used to encode a specific file, based on the occurrence of each data.

\begin{tabular}{|l|l||l|l||l|l|}
\hline Data & $\begin{array}{l}\text { Required Bits } \\
\text { (Code) }\end{array}$ & Data & $\begin{array}{l}\text { Required Bits } \\
\text { (Code) }\end{array}$ & Data & $\begin{array}{l}\text { Required Bits } \\
\text { (Code) }\end{array}$ \\
\hline \hline A & $3(110)$ & L & $5(00110)$ & R & $4(0110)$ \\
\hline C & $5(00010)$ & M & $4(0010)$ & S & $4(0111)$ \\
\hline D & $5(00011)$ & N & $5(00111)$ & T & $3(100)$ \\
\hline E & $3(111)$ & O & $4(0100)$ & - & $5(01011)$ \\
\hline I & $4(0000)$ & P & $5(01010)$ & space & $3(101)$ \\
\hline
\end{tabular}

Table 5: Bits required for each data. 


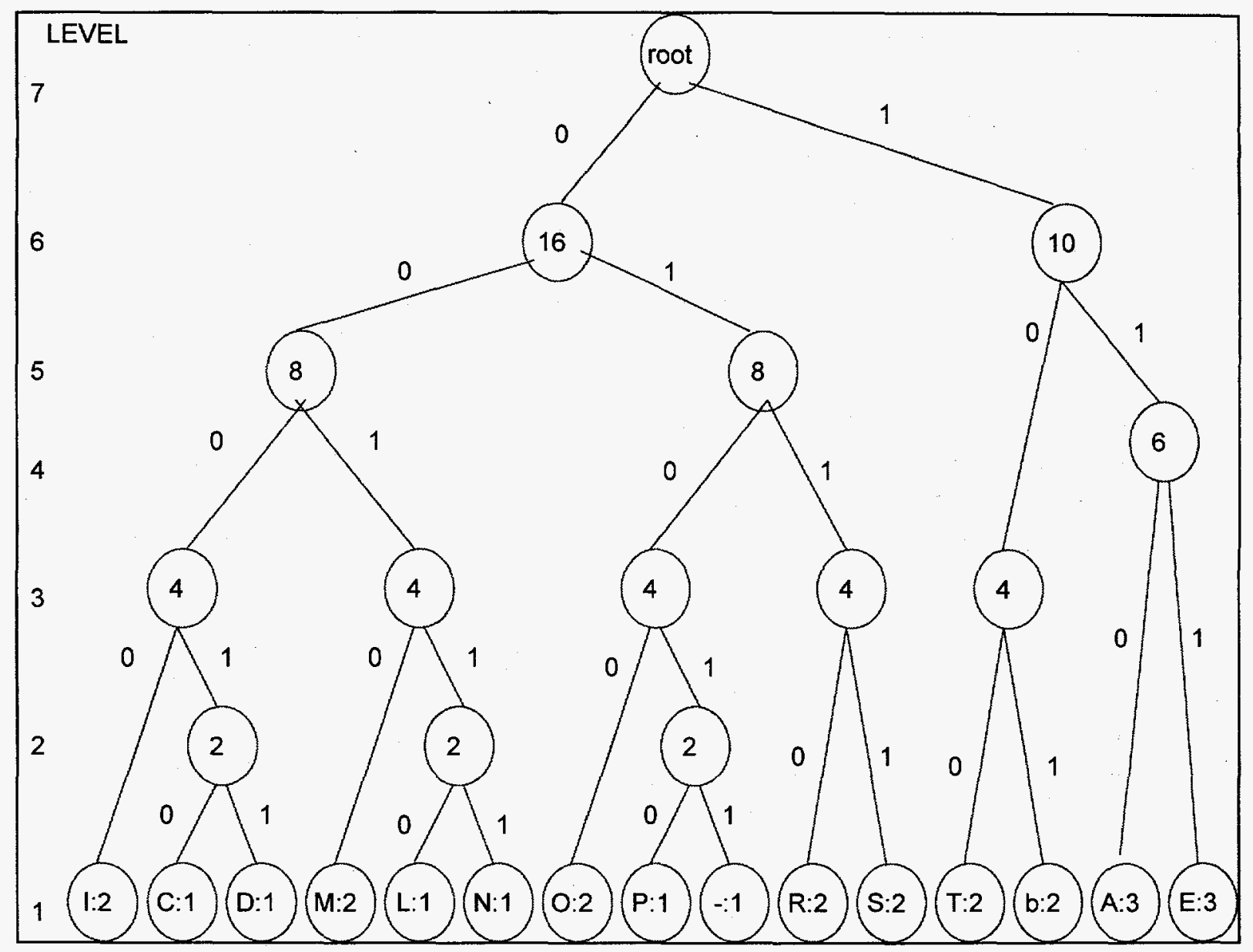

Figure 1: Tree used in example.

To determine the size of the compressed example file, multiply the occurrence of each data by the number of bits used to encode that data. In this example, the file length would be 100 bits. Since the phrase stored as ASCII used 208 bits, this scheme compressed the data $51.9 \%$.

One aspect about the data that is reflected in the compression ratio is the relative occurrence of the data. The more uniform the distribution is, the lower the compression ratio. For example, assume an even distribution between the 15 characters in the file. In this case the encoding tree becomes a straight binary tree. 14 of the characters require 4 bits to encode them and one character requires 3 bits. If each character occurs once, the compressed file becomes 59 bits long, compared to 120 bits for ASCII representation. This is a $50.8 \%$ compression ratio. On the other extreme, if one character occurs many more times than the other characters, the majority of the data in the file is encoded with relatively few bits. For example, if one of the 15 characters occurs 100 times and the other 14 occur only once each, the one character is encoded with just one bit, while 12 of the other 14 are encoded using 5 bits and the final two are encoded with 3 bits. This file is compressed to 166 bits. Compared 
to 912 bits for ASCII, this is a compression ratio of $81.8 \%$. As the data is further skewed toward the one character, the compression ratio approaches $7 / 8$ or $87.5 \%$. That is to say the compressed data approaches 1 bit for each data instead of 8 for ASCII. This is an upper bound for this compression scheme. Of course if the data file consisted of 1000 of the same character in a row, another encoding algorithm known as run-length-encoding (RLE) becomes very effective. However, RLE is beyond the scope of this paper.

The above discussion assumes the algorithm has access to all of the data before compression takes place. The data file is scanned completely, collecting statistical information on the data. This information is used to construct an encoding tree. Only then can the data be compressed. This is a problem in implementing the Huffman algorithm in a real-time system. A simple real-time system must handle the data byte by byte as it is collected. This precludes the use of the actual data in building the tree. Although adaptive algorithms may be used to change the tree on the fly, the algorithms tend to be cumbersome and are difficult to use on simple realtime systems.

Often the data that the real-time system is expected to collect during its operation is known approximately. Typically data is characterized during the design and test phase of the project. Exploiting this knowledge allows an representative Huffman tree to be constructed before the system is used in the field. This is advantageous to real-time systems as it allows the data to be compressed byte by byte as it is received.

Another advantage of constructing a tree prior to running the experiment is reduced overhead. Since the tree is known to the system developer, both the encoding and decoding routines may be hard coded with the tree. This eliminates the requirement of the encoding program to store the tree along with the data for the decoder to read. This results in further reducing the data storage requirements.

The representative tree can be constructed several ways. One way is to use the actual test data to build the tree as was done in the first example above. The data is scanned and a table of occurrence values constructed. The tree is built from there. This method would be particularly effective on highly predictable experiments. Another way to build a representative tree is to approximate the data set with a mathematical function. If the experimental data tended to follow some curve, a mathematical function could be used to generate data. The function would define the relative frequency of the data. The author believes the first method would be just as effective, if not more so, as the second method. However, in this paper, the method of using mathematical functions to approximate the data is examined. Showing the usefulness of using a representative curve implies the usefulness of using the actual curve. 


\section{Algorithm Implementation}

The algorithm was developed and tested on a PC based system. All of the software was written in $\mathrm{C}$. The compression and decompression programs follow the algorithm suggested in the above section, Theory, with one minor deviation. When the trees are constructed, a dummy node is added to the list of data. This node is only used once and signals the end of the file. It is included for diagnostic purposes and signals the correct ending to decoding. The software used is listed in the Appendixes.

The data used to develop and test the algorithm was gathered during environmental testing of a traveling railcar. This data is typical of accelerometer and environmental data. Figure 2 shows the raw data collected on the railcar. Notice the six distinct peaks in the curve. These curves are from the four distinct data sets gathered during the test. The composite data set consisted of the three accelerometer channels and one voltage channel. These data sets were interleaved as channel 1 , channel 2 , channel 3 , channel 4 , channel 1 , etc., repeating for the length of the experiment. A total of 256000 bytes were gathered, 64000 bytes for each of the four channels.

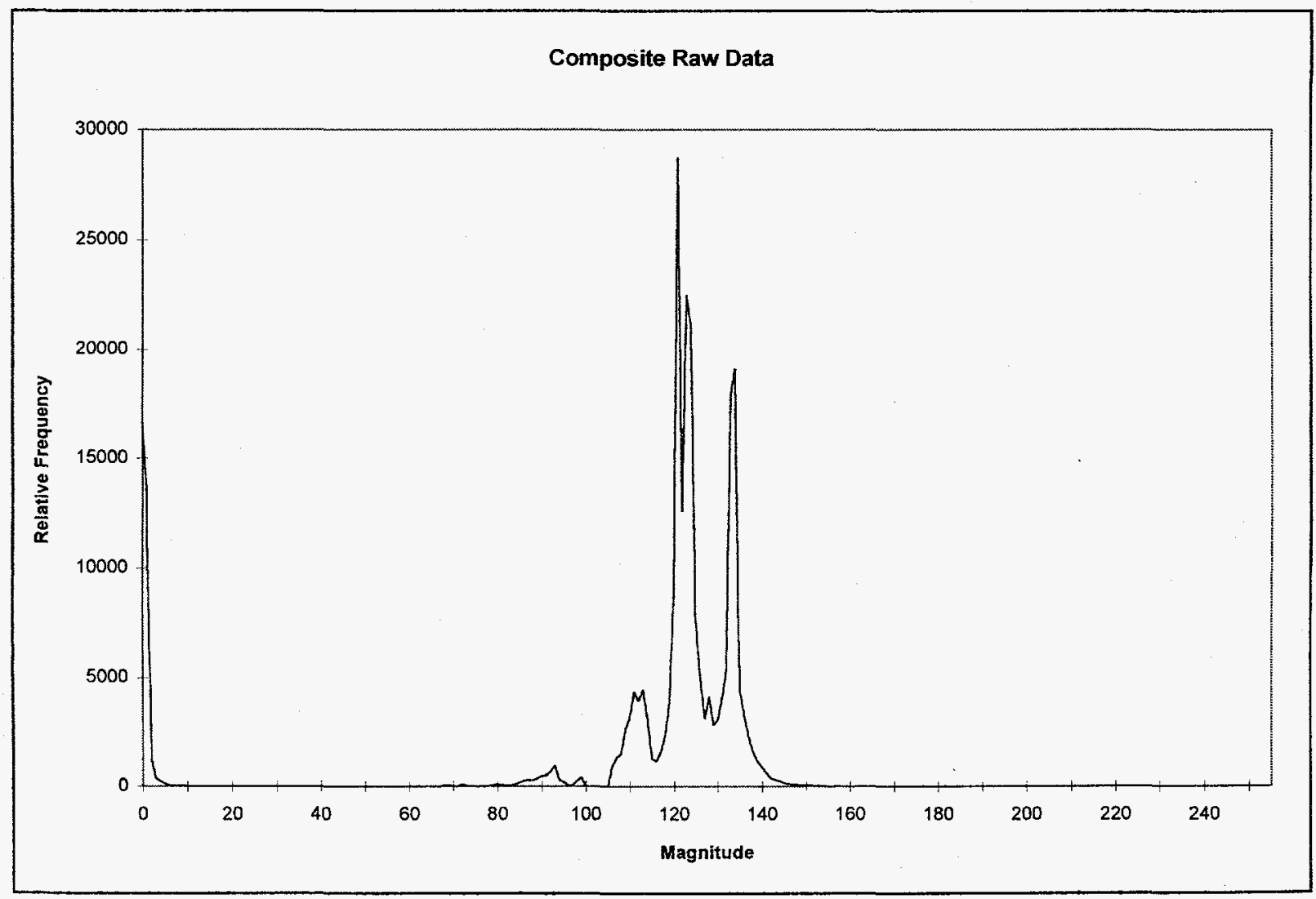

Figure 2: Composite data used in the algorithm development. 
These four data channels were subsequently separated out of the composite data set. Figure 3 , Figure 4, Figure 5, and Figure 6 show the data specific to channels 1, 2, 3, and 4, respectively.

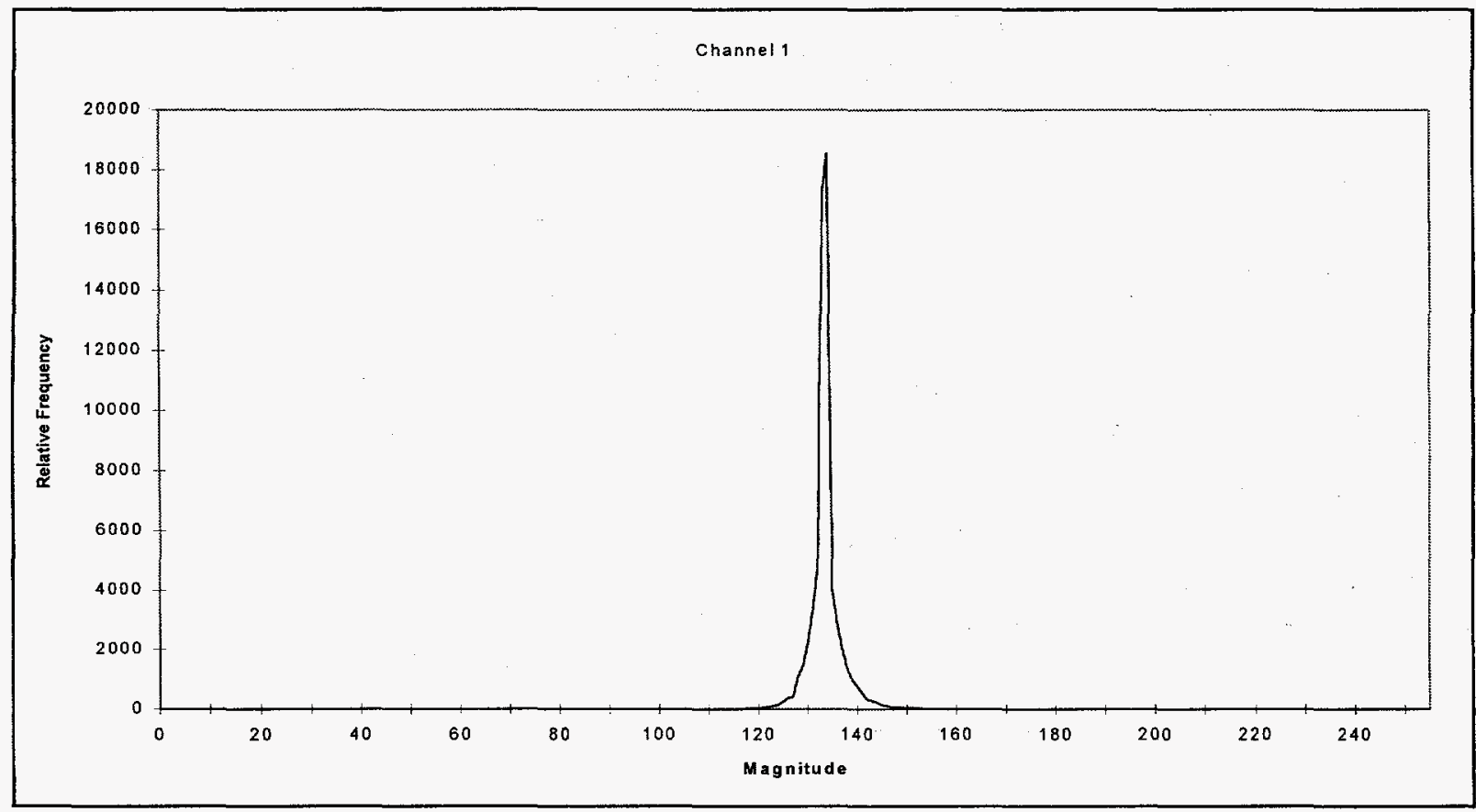

Figure 3: Accelerometer channel 1 data only.

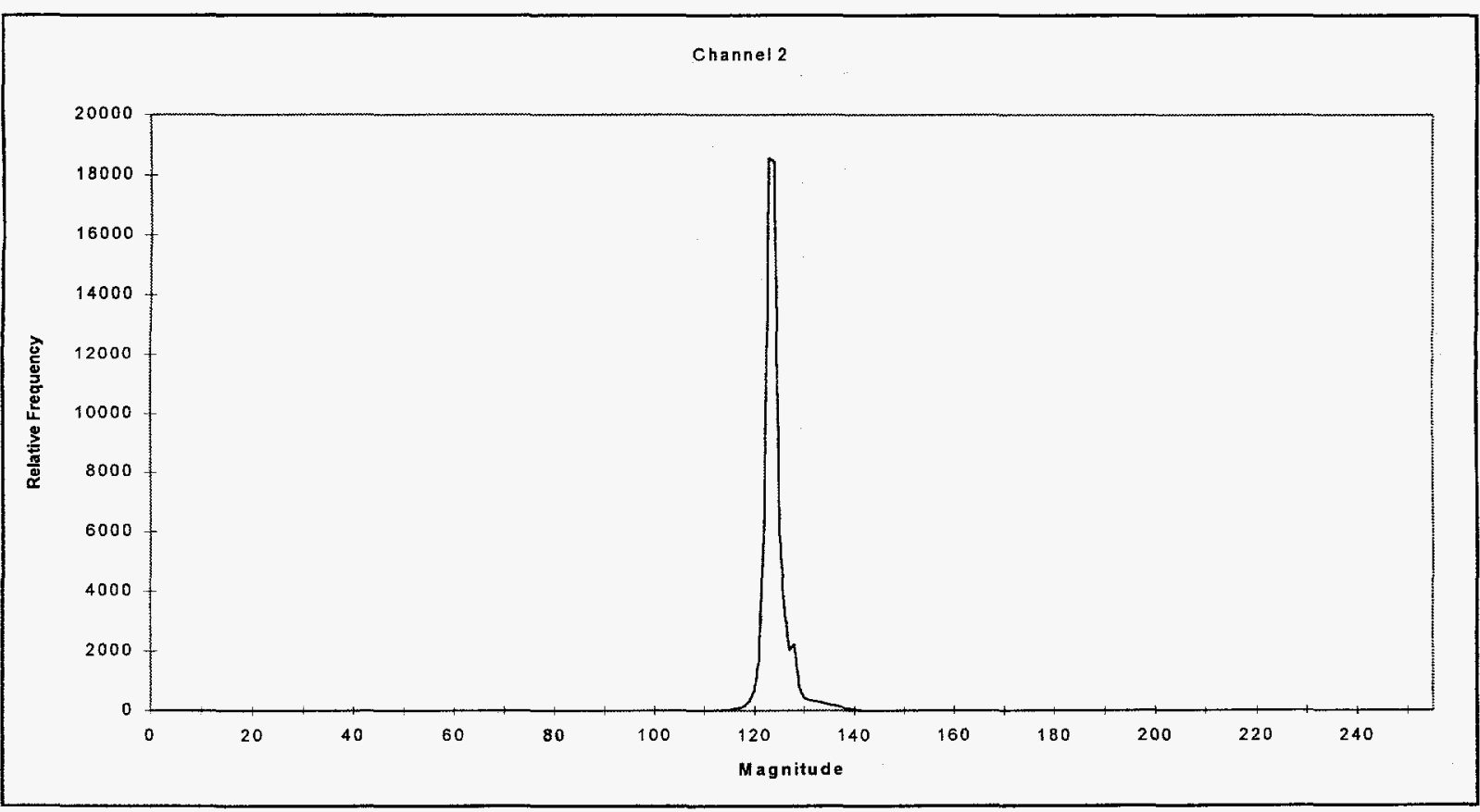

Figure 4: Accelerometer channel 2 data only. 


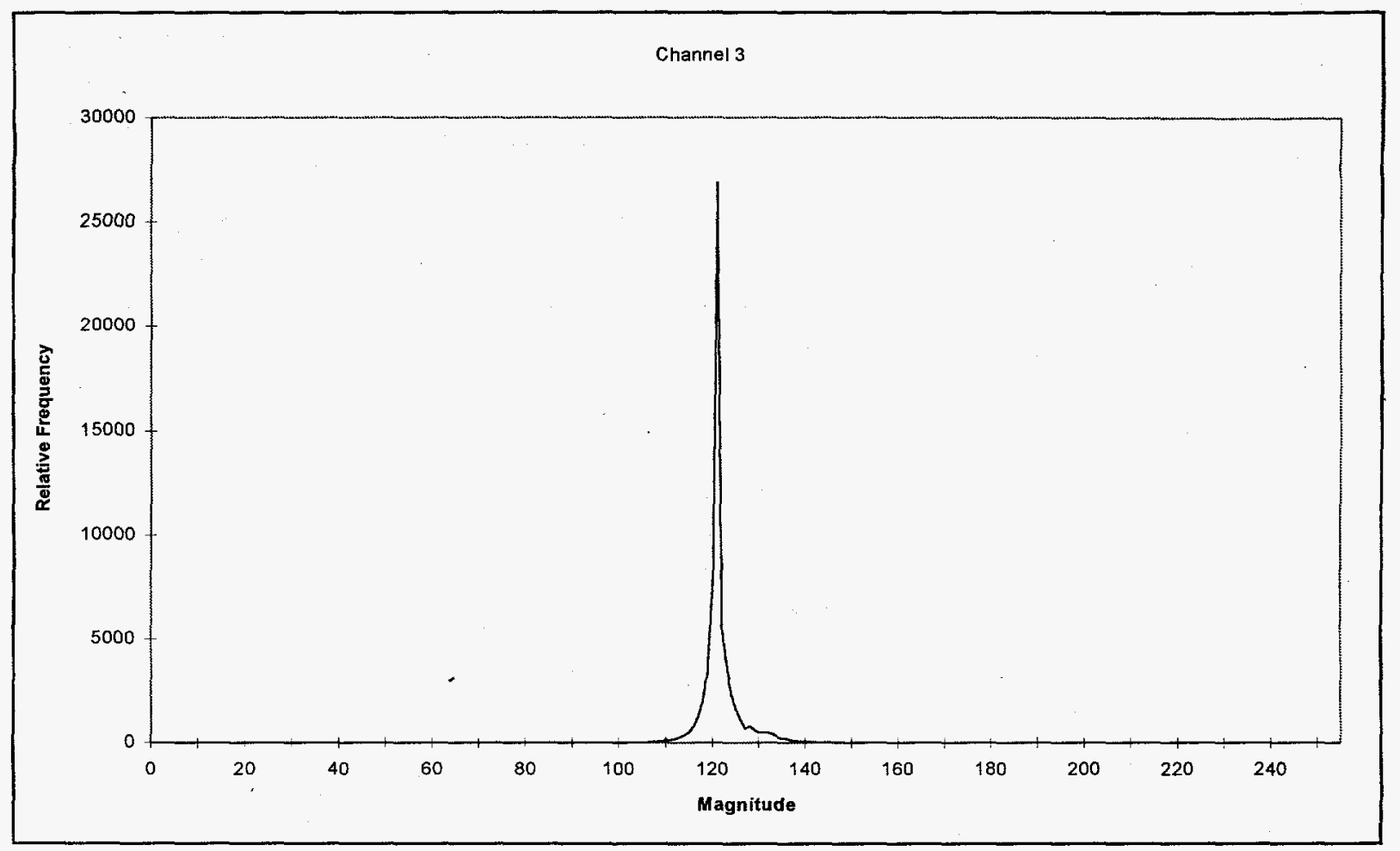

Figure 5: Accelerometer channel 3 data only.

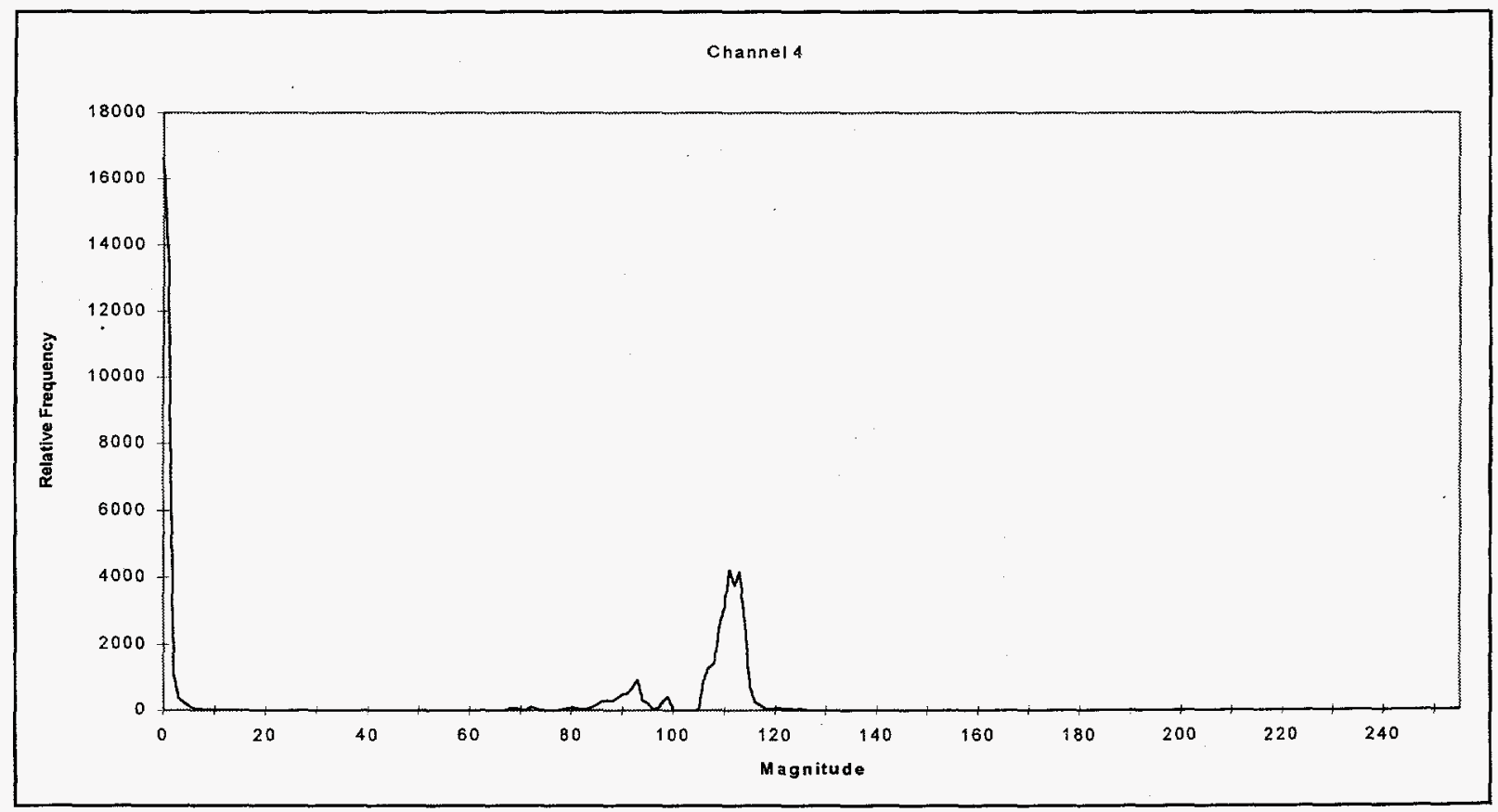

Figure 6: Supply voltage data only. 
Looking at the graphs for channels 1,2 , and 3 we see that the data is essentially localized to one area. The maximums for channels 1,2, and 3 occur at 134, 123, and 121 respectively. channel 4, however, has several local maximums in the graph. Specifically, channel 4 has a large maximum at 0 , a small maximum at 93 , and a medium maximum at 111 . Channel 4 poses a special problem since no one simple curve fits the data well. To compress channel 4 using representative curves requires more than one curve. Compression on the composite data also works better using more than one curve. In this paper we limit ourselves to a maximum of two simultaneous curves for simplicity. However comparing the use of one curve with two curves will emphasize the need for reasonable curve fitting. If possible, an entire data set could be used to directly generate the Huffman tree instead of trying to fit a curve to the data.

From these figures a representative curve was chosen to characterize the data. Rather than trying to match the curve exactly (which would change somewhat during the actual test), a slightly modified Gaussian distribution curve was used to approximate the data. The formula used to simulate the Gaussian distribution is

$$
f(x)=e^{\frac{-(x-c)^{2}}{o^{2}} * 8} * s
$$

where

$$
\begin{aligned}
& \mathrm{s}=\text { scale factor to size the output of the function ( } 255 \text { for } 8 \text { bit data) } \\
& \mathrm{c}=\text { the center, or mean, of the distribution } \\
& \mathrm{o}=\text { the offset, or deviation (essentially the standard deviation) }
\end{aligned}
$$

The program GEN_CODE.C implements a single curve using the above Gaussian distribution and generates the code tree. The program GEN_DUAL.C implements two Gaussian curves with different means and deviations. It also generates the code tree. The tree is incorporated into the compression program, ENCODE.C, through a $\mathrm{C}$ header file, CODES.H, which is used in compiling ENCODE.C. As a matter of convenience, both GEN CODE.C and GEN DUAL scale the Gaussian function(s) to fall within $0-255$. Also, all of the software assumes the data is 8 bit data and thus limited to a range of $0-255$. This range limit is superficial and the software could easily be modified to accommodate larger data words. GEN_CODE.C and $G E N \_D U A L$ compute the Gaussian function(s) for 256 points. These points are used to construct the tree found in CODES.H. Since ENCODE.C is compiled with the tree in place, it is ready to compress data byte by byte as the data is collected.

The decoding program, EXPAND.C, is not expected to run on the data acquisition system, and so the tree generating routines are still contained within the code. However, in order to decode the data properly, it must be compiled using the same Gaussian function(s) as ENCODE.C is compiled with. 
Due to a fixed scale factor of 255 for all of the tests, only the mean and deviation were varied. The fixed scale factor of 255 does not affect the end result. Since the Gaussian tree is constructed using the relative values of the data points, what the maximum value is makes no difference. These two values were optimized for each data set independently. Shown in Figure 7 and Figure 8 are the six curves used to study the effect of varying the Gaussian curve applied to channel 2. Similar curves were used on the other data sets. Table 6 lists the effects of several Gaussian curves on compressing channel 2.

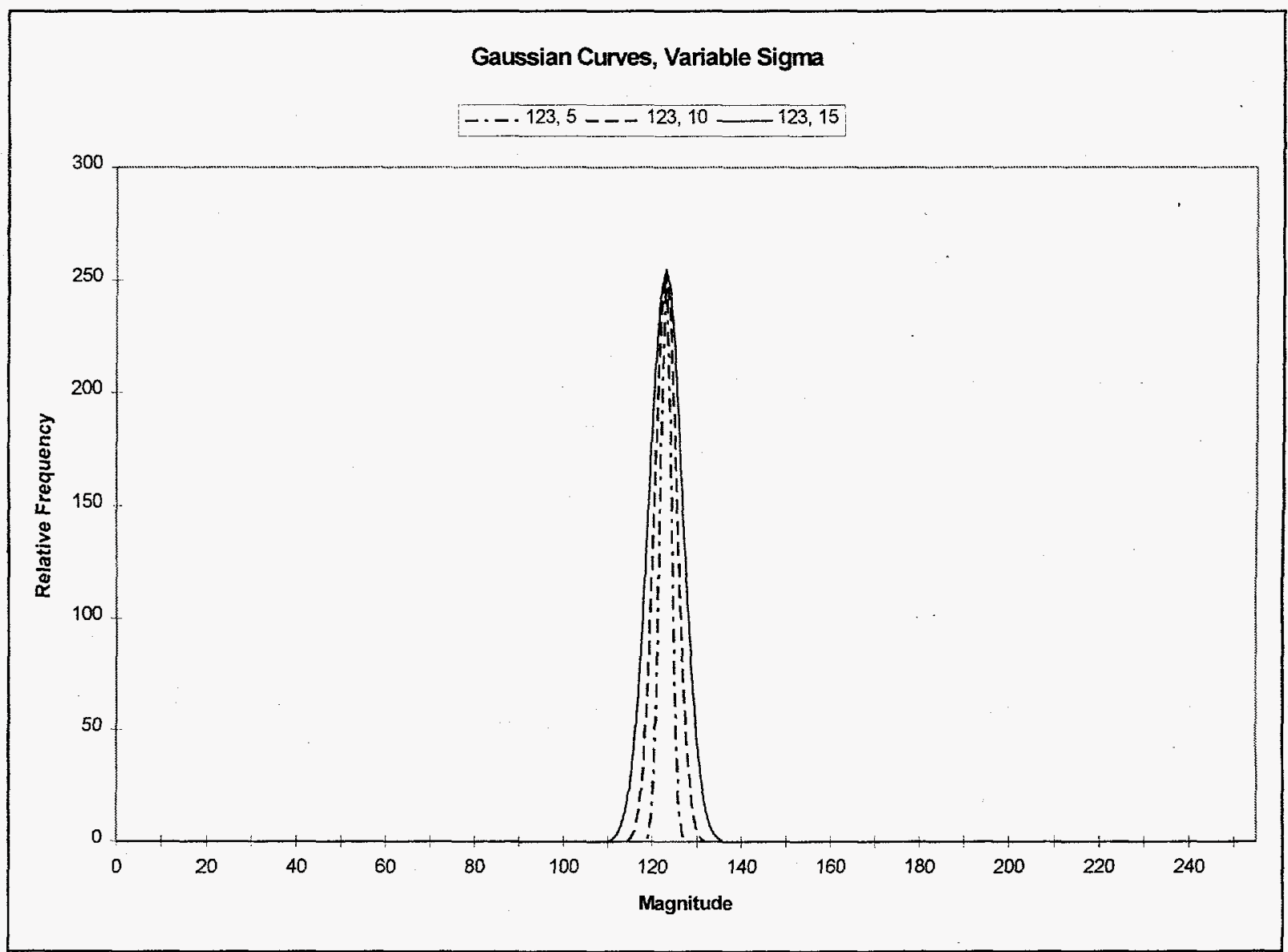

Figure 7: Three Gaussian curves with means of 123 and sigmas of 5,10 , and 15 . 


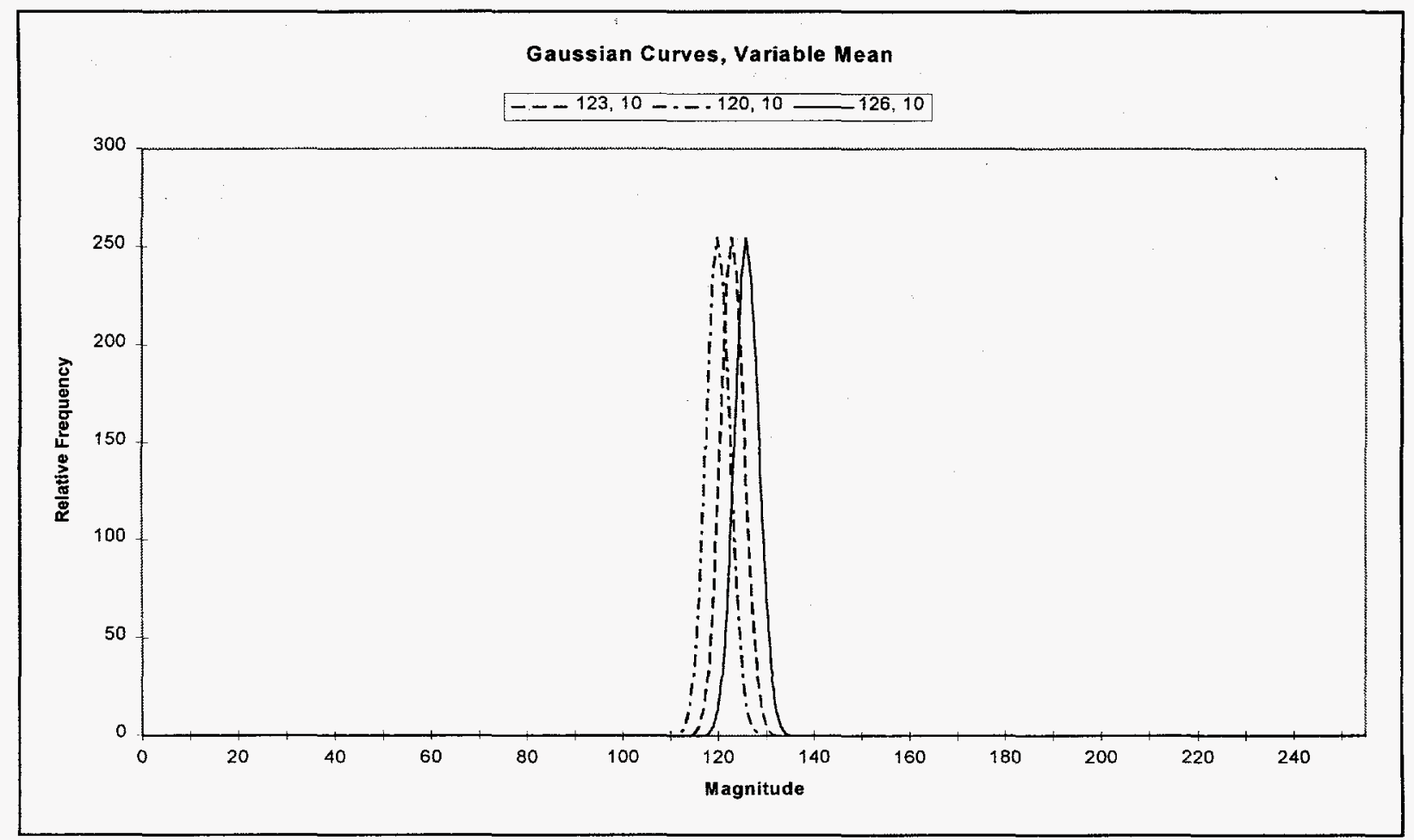

Figure 8: Three Gaussian curves with means of 120, 123, and 126 and sigmas of 10 .

\begin{tabular}{|l||l|l||l||l|}
\hline Mean & Sigma & Original Size & Compressed & \% Reduction \\
\hline \hline 123 & 5 & 64000 & 29776 & 53.5 \\
\hline 123 & 10 & 64000 & 28734 & 55.1 \\
\hline 123 & 13 & 64000 & 28804 & 55.0 \\
\hline 123 & 15 & 64000 & 29288 & 54.2 \\
\hline 123 & 25 & 64000 & 33224 & 48.1 \\
\hline 120 & 10 & 64000 & 41124 & 35.7 \\
\hline 126 & 10 & 64000 & 31462 & 50.8 \\
\hline
\end{tabular}

Table 6: Compression results for channel 2.

Table 6 suggests that the best compression ratio occurs when a mean of around 123 and a sigma of around 10 is used. Figure 9 shows a comparison of a Gaussian curve using these 
values and the channel 2 data. Notice that for comparison purposes, the number of occurrences of the channel 2 data was scaled to fall within $0-255$.

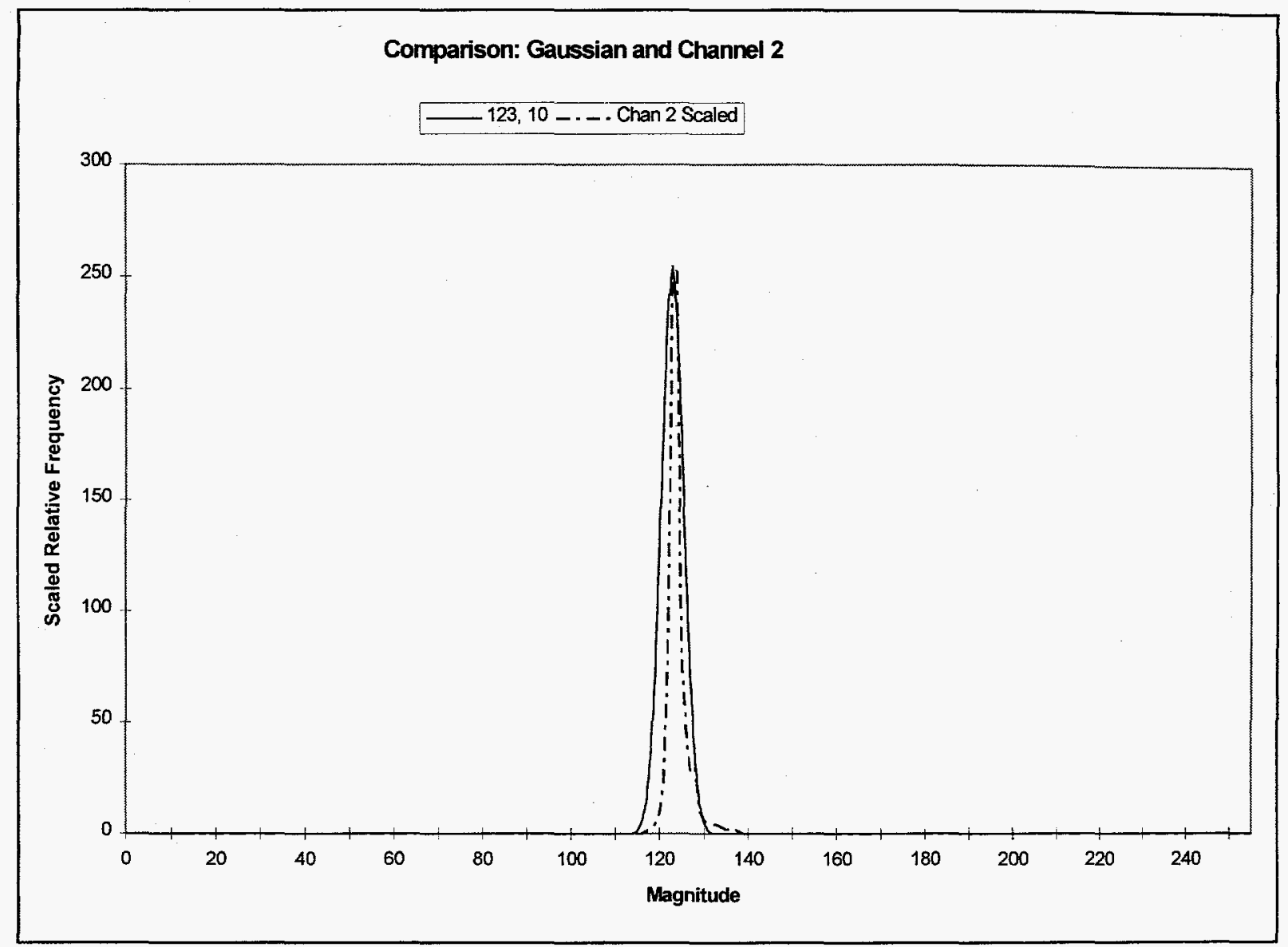

Figure 9: Gaussian curve yielding the greatest compression of the 5 on channel 2.

In the same way that channel 2 was tested, Table 7 lists the effects of several sets of single Gaussian curves on the composite data set. As expected, the compression ratios are nowhere near the compression ratios for channel 2. As suggested in Figure 2, a simple Gaussian distribution does not fit the curve very well, especially with the effects of channel 4 . To demonstrate the effects of using two curves, the composite data set was compressed using two Gaussian curves. Table 8: Comparison of single and dual function compression on the composite data set. compares the results of using two curves with the best result of using one curve. From this comparison we see the need to fit the representative curve to the data curve.

The other two accelerometer channels were also compressed individually using specific Gaussian curve parameters. The results of compressing these channels are shown in Table 9 along with the results of channels 2 and 4 . 


\begin{tabular}{|l||l|l||l||l|}
\hline Mean & Sigma & Original Size & Compressed & \% Reduction \\
\hline \hline 123 & 10 & 256000 & 233902 & 8.63 \\
\hline 123 & 20 & 256000 & 208220 & 18.7 \\
\hline 123 & 30 & 256000 & 198902 & 22.3 \\
\hline 123 & 33 & 256000 & 198122 & 22.6 \\
\hline 123 & 35 & 256000 & 198874 & 22.3 \\
\hline 123 & 40 & 256000 & 202180 & 21.0 \\
\hline 120 & 10 & 256000 & 245096 & 4.26 \\
\hline 125 & 10 & 256000 & 239874 & 6.30 \\
\hline
\end{tabular}

Table 7: Compression results for composite data using a single curve.

\begin{tabular}{|l||l||l||l||l|l||l|}
\hline Mean 1 & Sigma 1 & Mean 2 & Sigma 2 & Original Size & Compressed & \% Reduction \\
\hline \hline 123 & 30 & N/A & N/A & 256000 & 198902 & 22.3 \\
\hline 123 & 30 & 0 & 7 & 256000 & 172096 & 32.8 \\
\hline
\end{tabular}

Table 8: Comparison of single and dual function compression on the composite data set.

\begin{tabular}{|l||l||l||l||l||l|}
\hline Channel number & Mean & Sigma & Original Size & Compressed & \% Reduction \\
\hline \hline 1 & 133 & 13 & 64000 & 29964 & 53.2 \\
\hline 2 & 123 & 10 & 64000 & 28734 & 55.1 \\
\hline 3 & 121 & 14 & 64000 & 31012 & 51.5 \\
\hline 4 & 105 & 10 & 64000 & 63462 & 0.81 \\
\hline
\end{tabular}

Table 9: Channels 1, 2,3 and 4 compression results using a single curve. 
From Table 9 we see using a single curve to represent channel 4 fails miserably. Again, this is because from Figure 6 we see channel 4 appears as 3 distinct curves. Using two curves improves both the curve fitting and the compression ratio. The results are shown in Table 10.

\begin{tabular}{|l||l||l||l||l||l||l|}
\hline Mean 1 & Sigma 1 & Mean 2 & Sigma 2 & Original Size & Compressed & \% Reduction \\
\hline \hline 0 & 10 & 111 & 10 & 64000 & 36770 & 42.5 \\
\hline 0 & 7 & 111 & 10 & 64000 & 36068 & 43.6 \\
\hline 0 & 7 & 113 & 10 & 64000 & 37336 & 41.6 \\
\hline 0 & 7 & 111 & 15 & 64000 & 39380 & 38.5 \\
\hline
\end{tabular}

Table 10: Dual curve fitting for channel 4.

A final set of tests were run on a data set consisting of channels 1,2 , and 3 only. Channel 4 was removed from the composite data set. These three data channels, although separate and distinct curves, are close enough together to represent them with a single Gaussian curve. Table 11 shows the compression results using single Gaussian curves.

\begin{tabular}{|l||l||l|l|l|}
\hline Mean & Sigma & Original Size & Compressed & \% Reduction \\
\hline \hline 122 & 20 & 192000 & 129990 & 32.3 \\
\hline 126 & 20 & 192000 & 112768 & 41.3 \\
\hline 129 & 20 & 192000 & 119046 & 38.0 \\
\hline 126 & 30 & 192000 & 117254 & 38.9 \\
\hline
\end{tabular}

Table 11: Compression of channels 1, 2, and 3 using a single curve.

To get a feel for the significance of the performance of this fixed Huffman data reduction scheme, the compression results were compared to the compression results of PKZIP, version $2.04 \mathrm{~g}$, by PKWARE, Inc. The single function compression scheme demonstrated in this paper did perform quite well on individual accelerometer channels, since they had a simple, predictable curve to their data. Table 12 compares the compression ratios of the fixed Huffman routine assuming a single Gaussian function, with PKZIP. Notice the performance of the accelerometer channels 1,2, and 3. Again, these channels are well represented using a Gaussian curve. Also notice the poor results of channel 4 and the composite data, especially compared to PKZIP. These data sets are not well represented by a single Gaussian curve. 


\begin{tabular}{|l||l||l|}
\hline Data Set & PKZIP, Version 2 & Fixed Huffman \\
\hline \hline Channel 1 & $58 \%$ & $53 \%$ \\
\hline Channel 2 & $58 \%$ & $55 \%$ \\
\hline Channel 3 & $62 \%$ & $52 \%$ \\
\hline Channel 4 & $77 \%$ & $1 \%$ \\
\hline Composite Data & $60 \%$ & $23 \%$ \\
\hline
\end{tabular}

Table 12: Compression results comparison using a single Gaussian function.

Table 13 compares the compression ratios of the fixed Huffman routine on channel 4 and the composite data using two Gaussian functions to generate the tree. Although the ratios are only about half of PKZIP's ratios, the advantages of multiple curves is apparent.

\begin{tabular}{|l||l||l|}
\hline Data Set & PKZIP, Version 2 & Fixed Huffman \\
\hline \hline Channel 4 & $77 \%$ & $44 \%$ \\
\hline Composite Data & $60 \%$ & $33 \%$ \\
\hline
\end{tabular}

Table 13: Comparisons with PKZIP when using two functions to generate the tree.

The poor compression ratio of channel 4 as shown in Table 12 illustrates one last point. Representing channel 4 with a single function means more than half of the data is not represented. In the curve used here, only the data near 111 was covered by the curve. All of the data near zero and most of the data near 98 fell significantly outside of the curve. Still, with this poor representation, the data was not enlarged. This suggests how great the data mismatch would have to be before the compression scheme enlarges the data. This allows significant design tolerance errors in matching the actual test data.

\section{Conclusion}

The ideas presented in this paper represent a generalized method of quick, effective data compression. The algorithm is compact and consumes little CPU time. This algorithm is works well operating in simple embedded data acquisition systems. 


\section{Appendix}

The code for the algorithms used to demonstrate the ideas presented in this paper are listed below. All of the code was written in C. The code was compiled using Microsoft C Version 5.1 for DOS and was run on a PC platform.

The code listings in this appendix are listed in alphabetical order. The file name of the code and a brief description is given below.

DOS file name

Description

CODES.H

This is an example of a Huffman tree generated by GEN CODE.C. This file must be compiled into the encoding program ENCODE.C. The decoding program EXPAND.C must be altered to reflect the same curve function(s) as used here in order to decode the compressed data file correctly.

ENCODE.C This routine is used to compress the data file specified by the command line entry. It reads the Huffman tree defined in the $\mathrm{C}$ include file CODES.H.

EXPAND.C This program is used to decode the data file outputted by the program $E N C O D E . C$. It relies on the user to match the function(s) parameters used in the header file CODES.H, which was used in compiling ENCODE.C.

GEN_CODE.C This routine is used to convert one desired numerical function to the compression codes of a Huffman tree. The results of this program are stored as a header file which is used by ENCODE.C to compress a data file.

GEN_DUAL.C This routine is used to convert two desired numerical functions to the compression codes of a Huffman tree. The results of this program are stored as a header file which is used by ENCODE.C to compress a data file. 


\section{CODES.H}

\#define END_OF_STREAM 256

\#define Number- of BITS per sTORE 0x8000

int compress(unsigned int, unsigned int *);

int clean compress (unsigned int ${ }^{*}$ ):

unsigned int_mask5367 $=0 \times 8000$;

int rack5367 $=0$;

struct \{ unsigned int code; int code_bits; $\}$ code [] $=$ \{

$\{908,12\}$ \{ 2482,12$\}$

$\{909,12\}$,

\{ 2483, 12$\}$,

$\{225,10\}$

$\{910,12\}$,

$\{911,12\}$,

\{ 2432, 12$\}$,

$\{2484,12\}$,

$\{2485,12\}$,

$\{2486,12\}$,

$\{2487,12\}$

\{2434, 12$\}$,

$\{2488,12\}$,

\{ 2489, 12 \}

\{ 2435, 12 \},

\{ 2490, 12$\}$,

$\{2491,12\}$,

$\{2492,12\}$,

$\{2493,12\}$,

$\{2494,12\}$,

\{ 2495, 12 \}

$\{2496,12\}$,

\{ 2497, 12 \},

\{ 2498, 12$\}$,

$\{2499,12\}$,

$\{2500,12\}$,

\{ 2501, 12 \}

$\{2502,12\}$,

\{ 2503, 12 \},

\{ 2504, 12 \}

\{ 2505, 12$\}$,

( 2506, 12 \}

\{ 2507, 12 \},

\{ 2508, 12$\}$

\{ 2509, 12 \},

\{ 2510,12$\}$

\{ 2511, 12$\}$,

\{ 2512,12$\}$,

$\{2513,12\}$,

\{ 2514, 12 \},

$\{2515,12\}$,

$\{2516,12\}$,

\{ 2517, 12$\}$,

$\{2518,12\}$,

$\{2519,12\}$,

$\{2520,12\}$,

\{ 2521, 12$\}$,

$\{2522,12\}$,

$\{2523,12\}$

\{ 2524, 12 \},

$\{2525,12\}$,

$\{2526,12\}$,

\{2527, 12$\}$

\{ 2528, 12 \}

$\{2529,12\}$

\{ 2530,12$\}$,

\{ 2531,123 ,

$\{2532,12\}$,

\{ 2533,12$\}$,

\{ 2534,12$\}$

$\{854,10\}$

\{206, 9$\}$,

[57,8\},

[ 214, 8\},

\{29, 7\},

$\{0,6\}$,

$\{15,6\}$,

$\{58,6\}$,

$\{1,5\}$

$\{13,5\}$,

$\{27,5\}$,

\{30, 5$\}$,

$\{1,4\}$,

\{4, 4 \},

$\{7,4\}$,

\{10, 4\},

( 12, 43,

\{ 11,4$\}$,

[8, 4 \},

$\{5,4\}$,

\{ 2,4 \},

$\{31,5\}$,

$\{28,5\}$,

$\{18,5\}$,

$\{6,5\}$,

$\{59,6\}$,

\{ 24,6$\}$,

$\{1,6\}$,

$\{50,7\}$,

$\{215,8\}$,

$\{102,8\}$,

\{ 207,9$\}$

$\{855,10\}$,

$\{226,10\}$

\{ 2536, 12$\}$,

\{ 2537, 12 \},

$\{2538,12\}$,

$\{2539,12\}$,

$\{2540,12\}$,

\{ 2541, 12 \},

$\{2542,12\}$,

$\{2543,12\}$,

( 2544, 12 \},

\{ 2545, 12 \}

$\{2546,12\}$,

\{ 2547, 12 \},

$\{2548,12\}$,

$\{2549,12\}$,

$\{2550,12\}$,

\{ 2551, 12 \},

\{ 2552, 12 \},

\{ 2553,12 \}

\{2555, 12$\}$,

\{2556, 12$\}$,

\{ 2557, 12 \},

$\{2558,12\}$,

\{ 2559, 12$\}$,

[3328, 12$\}$,

$\{3329,12\}$,

$\{3330,12\}$,

$\{3331,12\}$,

$\{3332,12\}$,

$\{3333,12\}$,

$\{3334,12\}$,

$\{3335,12\}$,

$\{3336,12\}$,

$\{3337,12\}$,

$\{3338,12\}$,

$\{3339,12\}$,

$\{3340,12\}$,

$\{3341,12\}$.

\{ 3342, 12 \},

$\{3343,12\}$,

$\{3344,12\}$,

$\{3345,12\}$,

$\{3346,12\}$,

$\{3347,12\}$

\{ 3348,12$\}$,

$\{3349,12\}$,

$\{3350,12\}$,

$\{3351,12\}$,

\{ 3352, 12 \},

$\{3353,12\}$,

[ 3354, 12 \},

\{ 3355, 12$\}$,

\{ 3356,12 \}

\{ 3357,12 \},

$\{3358,12\}$,

$\{3359,12\}$,

$\{3360,12\}$,

$\{3361,12\}$,

\{3362, 12 \}

$\{3363,12\}$,

\{ 3364, 12$\}$,

$\{3365,12\}$,

( 3366, 12 \},

\{ 3367,12$\}$,

$\{3368,12\}$,

$\{3369,12\}$,

\{ 3370,12$\}$,

\{ 3371,12$\}$,

\{ 3372, 12$\}$,

( 3373,12 )

( 3374,12$\}$,

( 3375,12$)$,

( 3376,12 ),
[ 3377,12 \} $\{3378,12\}$, \{ 3379,12$\}$, ( 3380,12$\}$, $\{3381,12\}$, $\{3382,12\}$, $\{3383,12\}$, \{ 3384,12$\}$, \{ 3385,12$\}$, ( 3386,12 \}, ( 3387,12 ), ( 3388,12 ), ( 3389,12 ), \{ 3390,12$\}$, \{ 3391,12 \}, \{ 3392, 12 \}, $\{3393,12\}$, [3394, 12 \}, $\{3395,12\}$, \{ 3396, 12 \}, [ 3397, 12 \}, \{ 3398,12$\}$, $\{3399,12\}$, (3400, 12 \}, $\{3401,12\}$, \{3402, 12$\}$, ( 3403,12$\}$, \{ 3404,12 \}, \{ 3405,12$\}$, ( 3406,12 ), \{ 3407,12 \}, ( 3408,12$\}$, \{ 3409,12 \}, ( 3410,12$)$, \{ 3411,12 \}, $\{3412,12\}$, \{ 3413,12$\}$, $\{3414,12\}$, (3415, 12 ), $\{448,11\}$, \{ 449,11$\}$ 3 ; 


\section{ENCODE.C}

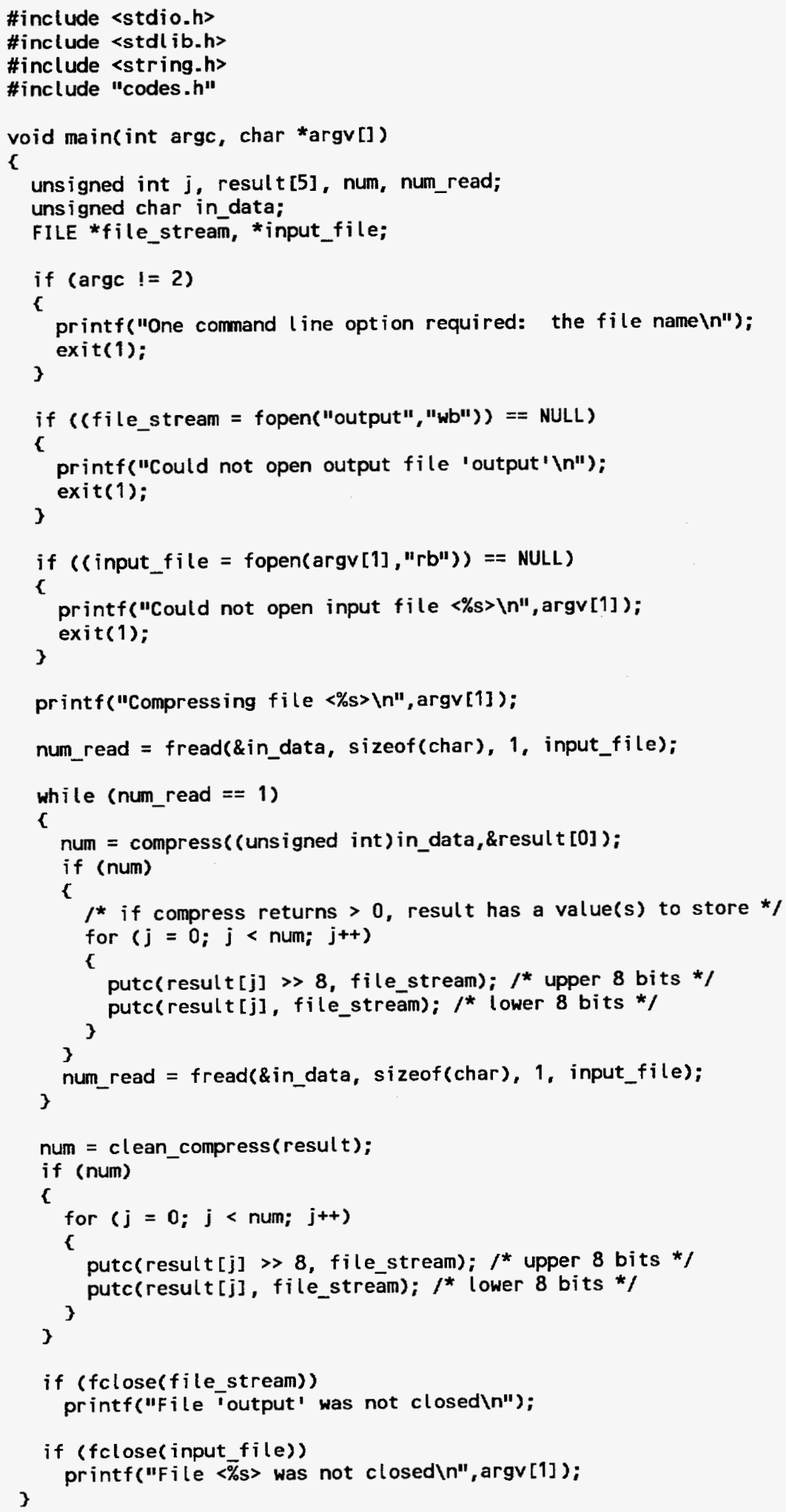


int compress(unsigned int in_number, unsigned int *out_number)

c

unsigned long temp_mask;

unsigned char out_count $=0$;

temp_mask $=1 L \ll($ code [in_number]. code_bits -1$)$;

whilé (temp_mask $!=0$ )

[

if (temp mask \& code [in_number] -code)

$\operatorname{rack} 5 \overline{3} 67 \mid=\operatorname{mas} k 536 \overline{7}$

mask5367 >>= 1

if ( $($ mask5367 $==0$ )

r

out_number [out_count++] = _rack5367;

$-\operatorname{rack5367}=0$;

3

_mask5367 = Number_of_BITS_per_sTORE;

3

temp_mask $>=1$;

3

return (out_count):

int clean_compress(unsigned int *out_number)

r

unsigned char out_count;

out_count $=$ (unsigned char)compress( (unsigned int)END_OF_STREAM, out_number);

if (_mask5367 ! = Number_of_BITS_per_sTORE)

\{

out number [out count++] = rack5367;

_rack5367 = 0;

3

mask5367 = Number_of_BITS_per_sTORE;

\}

return (out_count); 


\section{EXPAND.C}

\#include <stdio.h>

\#include <stdlib.h>

\#include <math. $h>$

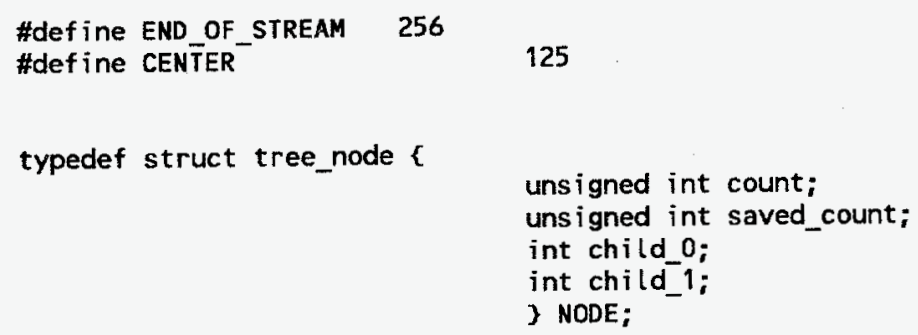

NODE nodes [514];

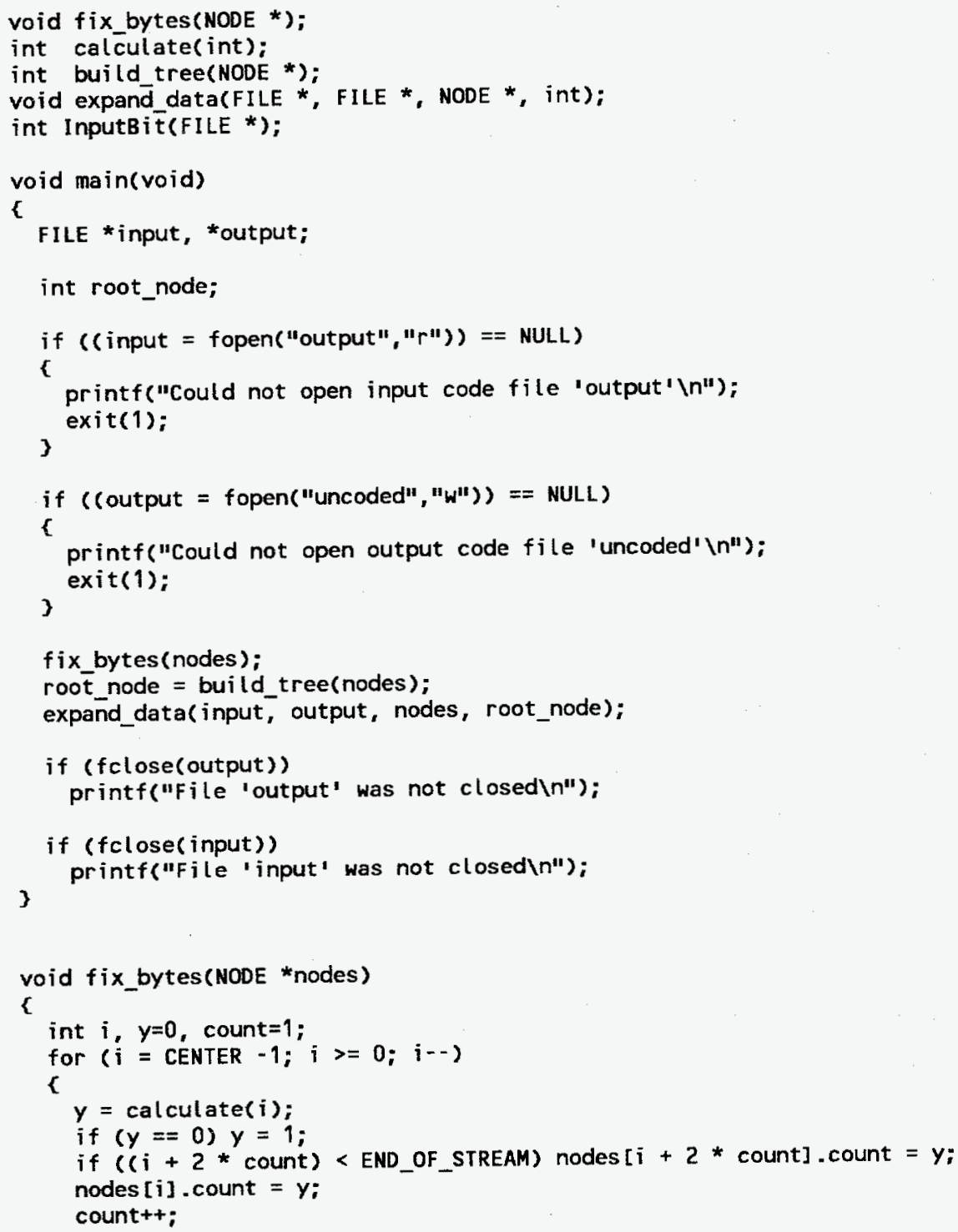




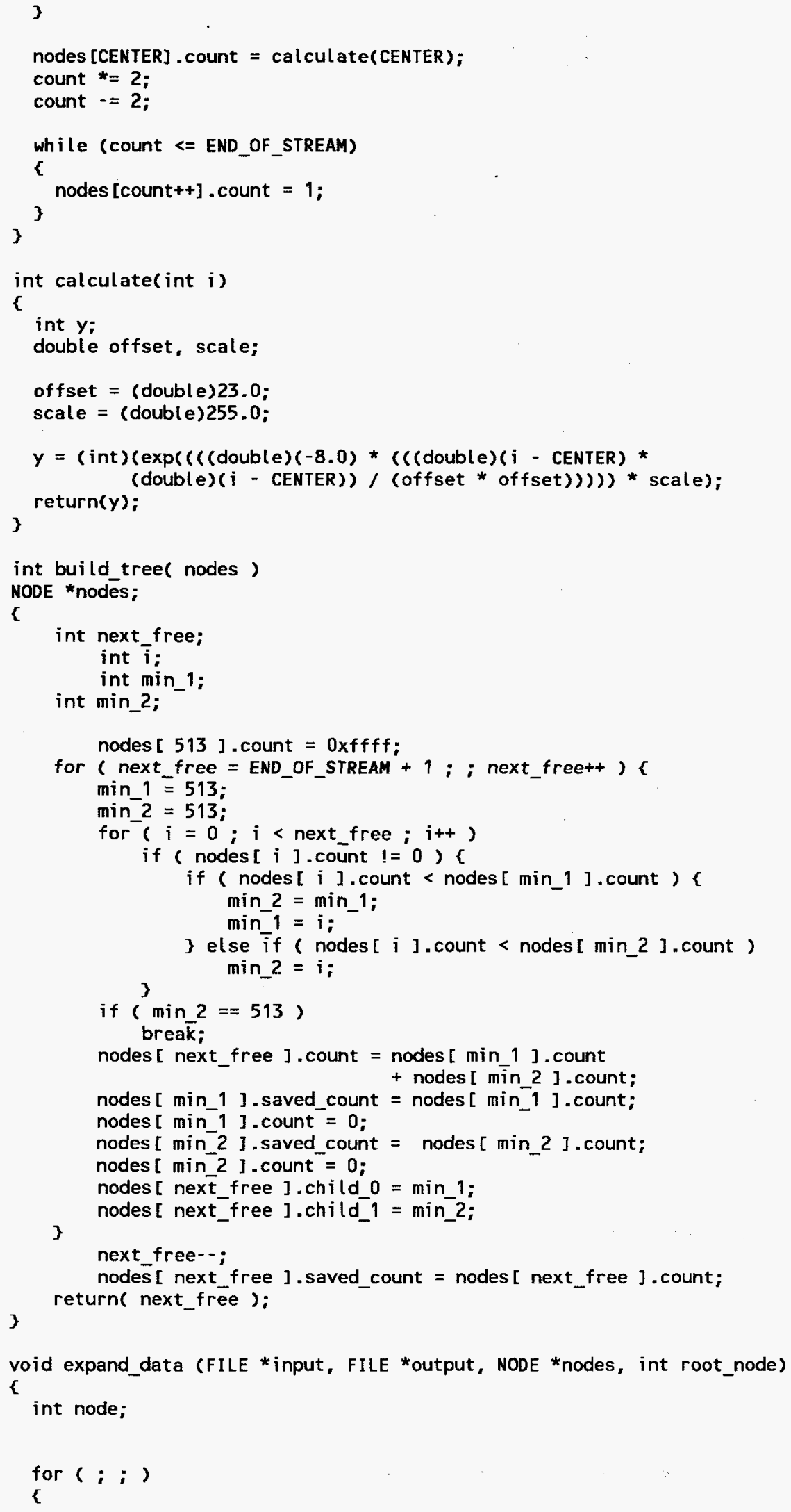




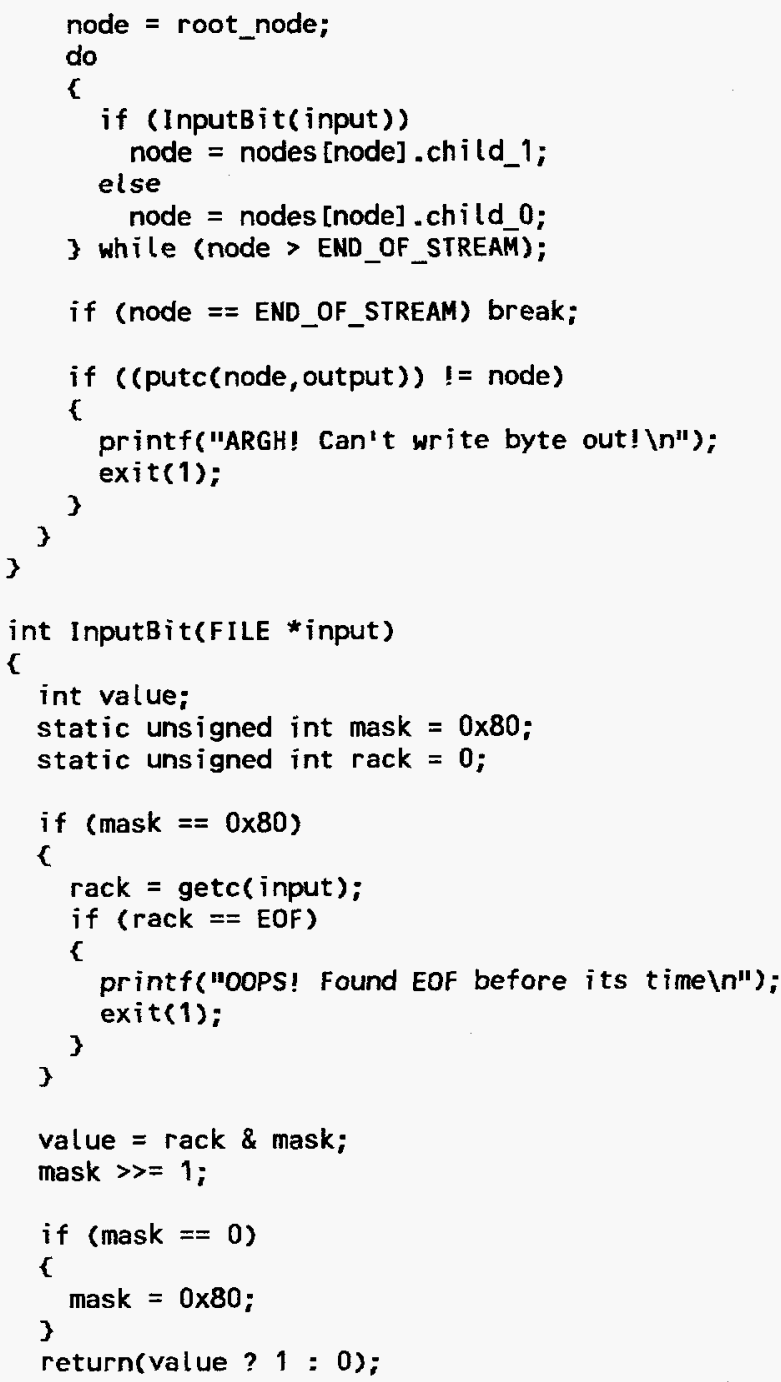




\section{GEN_CODE.C}

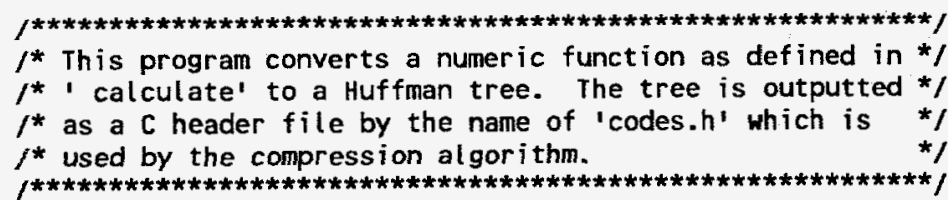

\#include <stdio.h>

\#include <stdlib.h>

\#include <math.h>

\#define END_OF_STREAM 256

**** The following defines determine the Gaussian curve */ \#define SCALE

255

typedef struct tree_node [

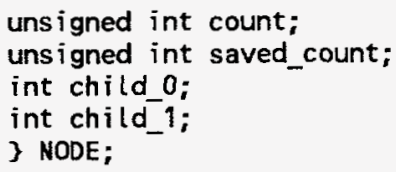

typedef struct code \{

unsigned int code;

int code bits;

3) CODE;

NODE nodes [514]

CODE codes $[257]$;

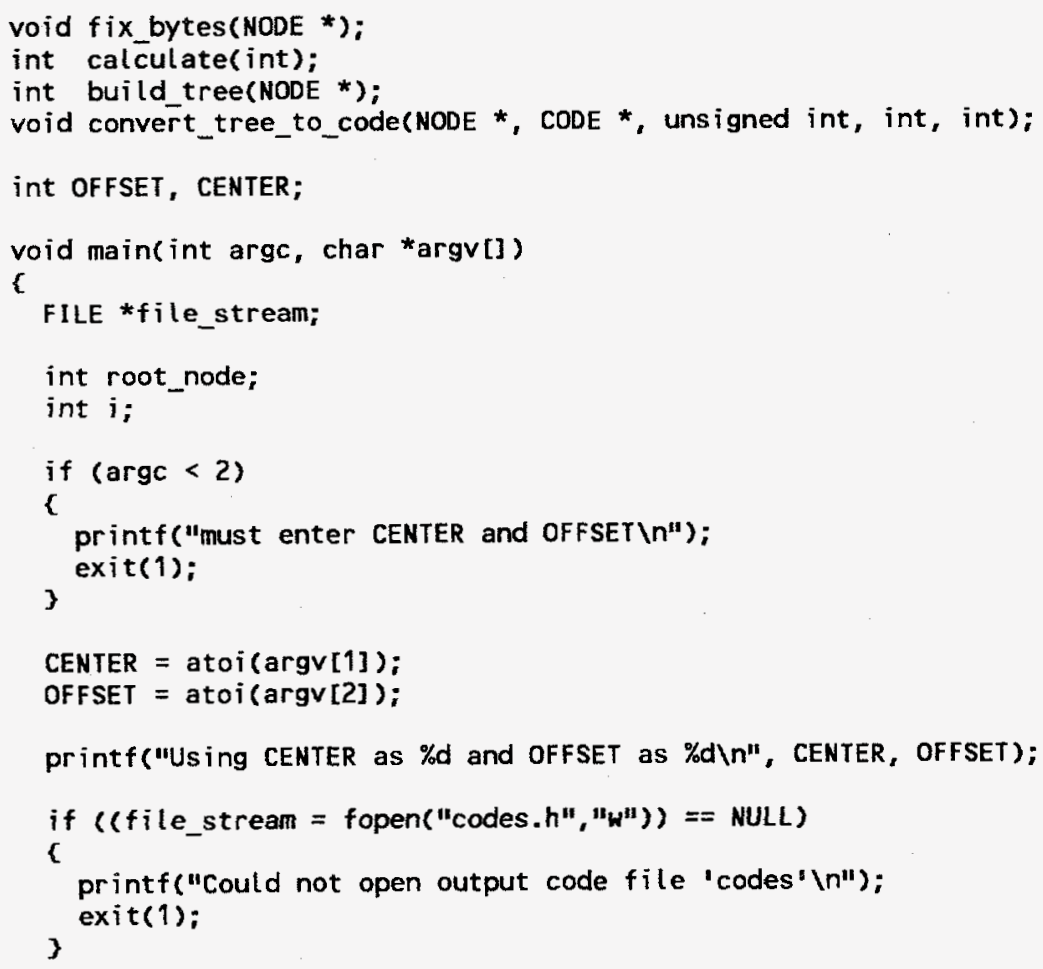




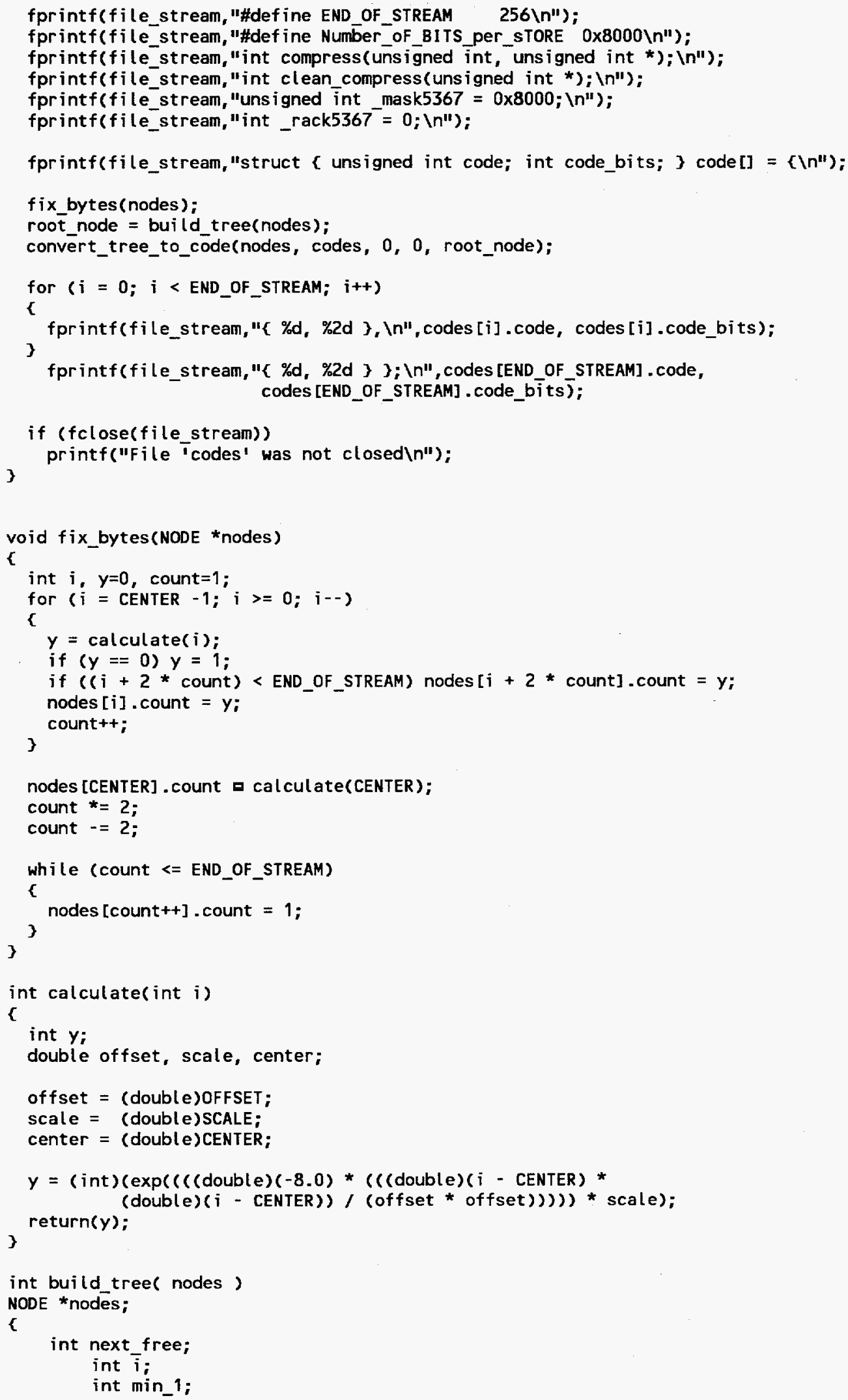


int min_2;

nodes $[513]$.count $=0 x f f f$;

for ( next_free = END_OF_STREAM + 1 ; ; next_free++ ) (

$\min 1=513$

$\min 2=513$

for $(i=0 ; i<$ next free : $i++)$

if ( nodes [ $i]$.count $!=0)($

if ( nodes $[i]$.count $<$ nodes $[\min 1]$.count )

min_2 = min_1;

$\min 1=i$.

\} else if ( nodes [ i ].count < nodes [ min_2 ] .count)

3 min_2 $=\mathrm{i}$;

if $(\min 2=513)$

break;

nodes $[$ next_free $]$.count $=$ nodes $[\min 11]$.count

+ nodes [ min_2 ] count:

nodes $[$ min_1 $]$. saved_count $=$ nodes $[$ min_1 $]$. count;

nodes [ min 1$]$. count $=0$;

nodes $[$ min_2 $]$.saved_count $=$ nodes $[$ min_2 $]$.count

nodes [ min_2 ]. count $=0$;

nodes [ nex $\bar{t}$ free ].child_0 $=\min \_1$;

3

nodes [ next_free $].$ child_1 $=$ min_2;

\}

next free-:-:

nodes $[$ next_free $]$.saved_count $=$ nodes $[$ next_free $]$.count; return( next_free );

void convert tree_to_code( nodes, codes, code_so_far, bits, node) NODE *nodes;

CODE *codes;

unsigned int code_so_far;

int bits:

int node;

c

if ( node <= END OF STREAM ) \{

codes $[$ node $]$.code $=$ code_so_far:

codes [ node ].code_bits = bits;

return;

3

code_so_far $\ll=1$;

bits+r;

convert_tree_to_code( nodes, codes, code_so_far, bits, nodes [ node ].child $0^{-}$);

convert_tree_to_code( nodes, codes, code_so_far | 1 ,

3

bits, nodes [ node $\overline{]} . c \bar{h} i l d \_1$ ); 


\section{GEN DUAL.C}

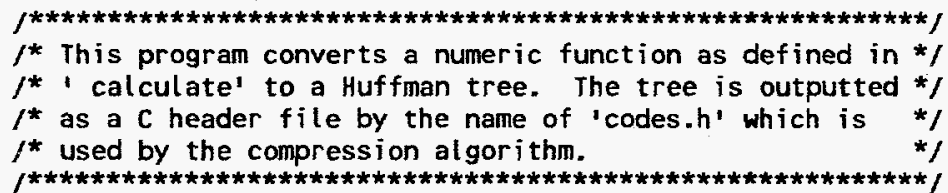

\#include <stdio.h>

\#include <stdl ib.h>

\#include <math. $h\rangle$

\#define END_of_STREAM 256

$f * * * *$ The following defines determine the Gaussian curve */

\#define SCALE

255

typedef struct tree_node $<$

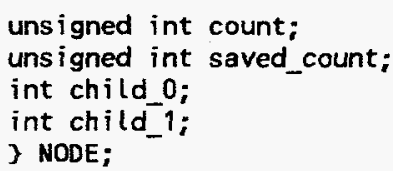

typedef struct code \{

$$
\begin{aligned}
& \text { unsigned int code; } \\
& \text { int code_bits; } \\
& 3 \text { CODE; }
\end{aligned}
$$

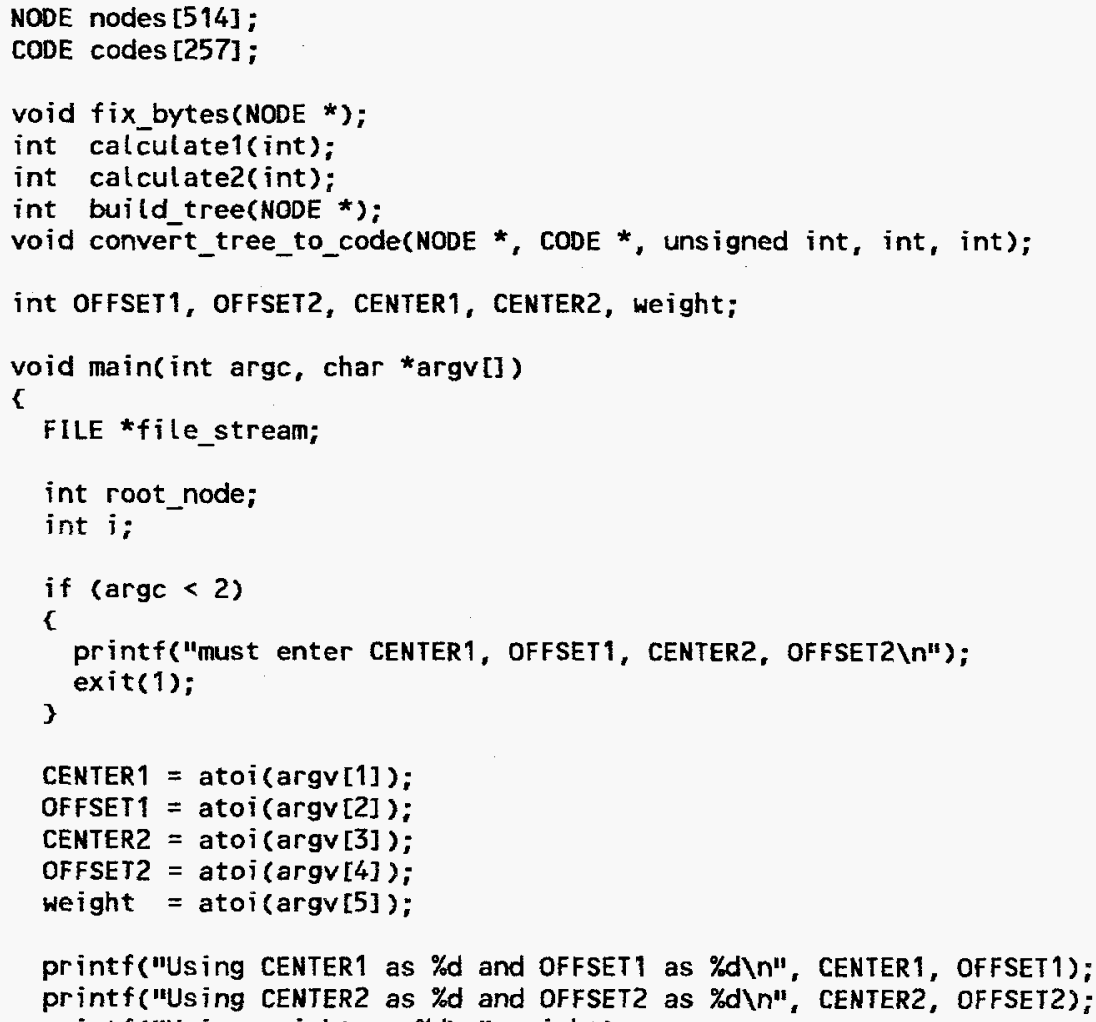

printf "Using CENTER1 as \%d and OFFSET1 as \%d\n", CENTER1, OFFSET1): printf("Using CENTER2 as \%d and OFFSET2 as \%dIn", CENTER2, OFFSET2); printf ("Using weight as \%dln", weight); 


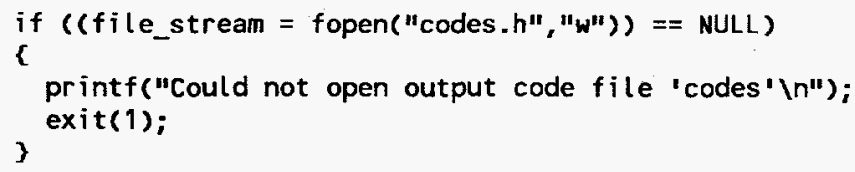




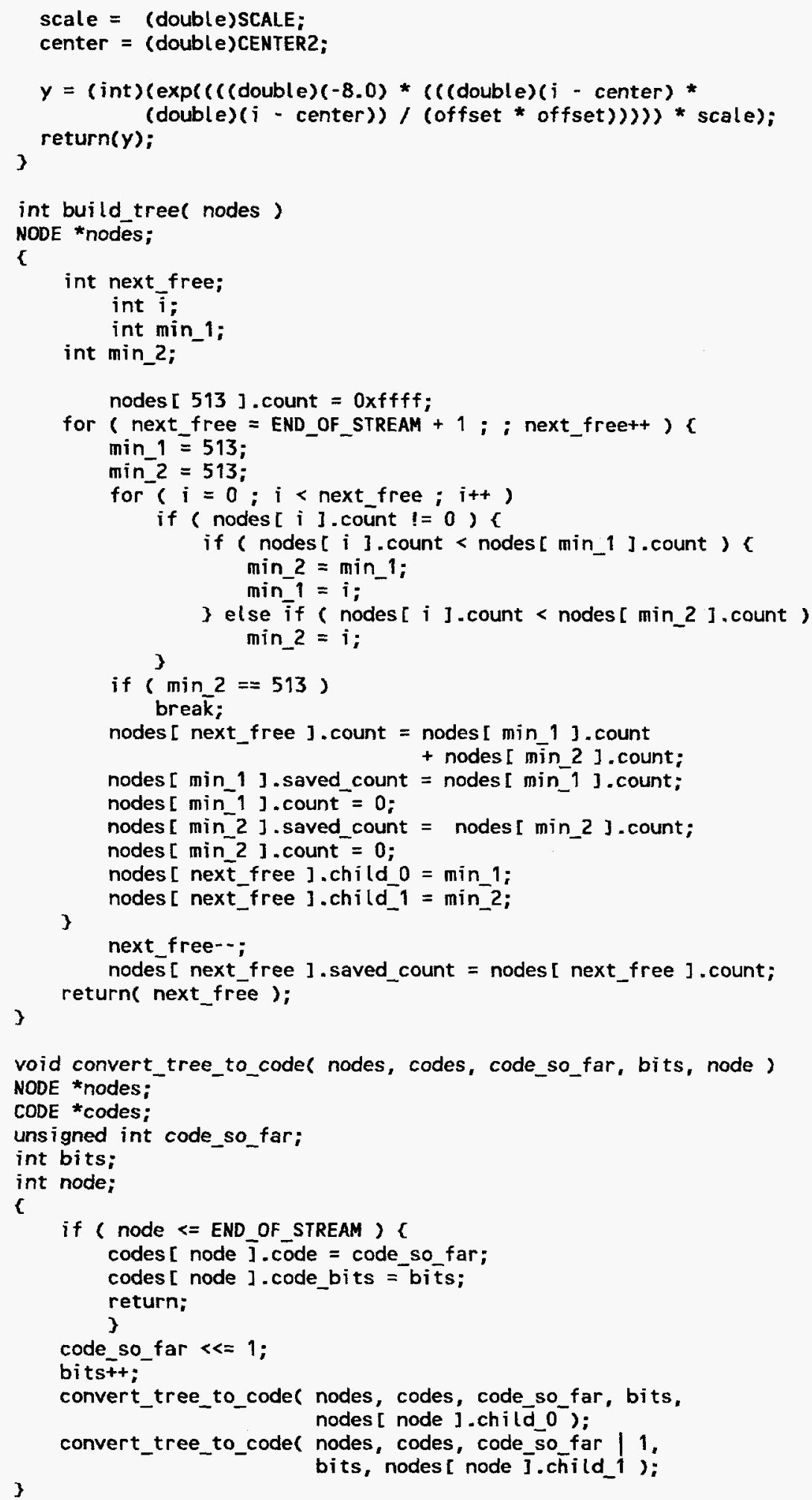




\section{DISTRIBUTION:}

$\begin{array}{lll}\text { Copies } & \text { Mail Stop } & \text { Recipient } \\ 1 & 0985 & \text { J. H. Stichman, 2600 } \\ 1 & 0987 & \text { D. E. Ryerson, 2664 } \\ 10 & 0987 & \text { R. E. Kidner, 2664 } \\ 1 & 9018 & \text { Central Technical Files, 8523-2 } \\ 5 & 0899 & \text { Technical Library, 13414 } \\ 1 & 0619 & \text { Technical Publications, 12613 } \\ 2 & 0100 & \text { Document Processing for DOE/OSTI, 7613-2 }\end{array}$

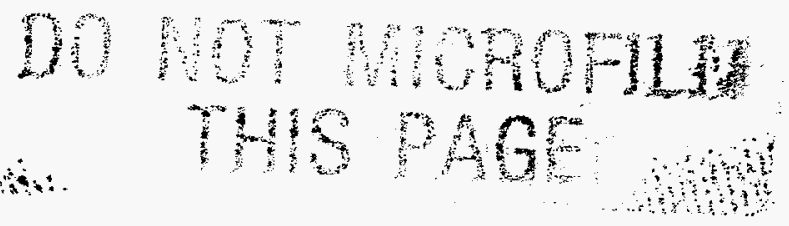

